\title{
PRAGMATIC LANGUAGES WITH UNIVERSAL GRAMMARS
}

\author{
PENÉLOPE HERNÁNDEZ, AMPARO URBANO, AND JOSÉ E. VILA
}

\begin{abstract}
This paper shows the existence of an equilibrium pragmatic Language with a universal grammar as a coordination device under communication misunderstandings. Such a language plays a key role in achieving efficient outcomes in those Sender-Receiver games where there may exist noisy information transmission. The Language is pragmatic in the sense that the Receiver' best response depends on the context, i.e, on the payoffs and on the initial probability distribution of the states of nature of the underlying game. The Language has a universal grammar because the coding rule does not depend on such specific parameters and can then be applied to any Sender-Receiver game with noisy communication.
\end{abstract}

Keywords: grammar, pragmatic language, prototypes, separating equilibria.

JEL Classification: C61, C73, D82.

\footnotetext{
Departament of Economic Analysis and ERI-CES. University of Valencia. Facultad de Economia, Campus dels Tarongers. 46022 Valencia (Spain). e-mails: penelope.hernandez@uv.es; amparo.urbano@uv.es and jose.e.vila@uv.es. The authors thank both the Spanish Ministry of Science and Technology and the European Feder Founds for financial support under project SEJ2007-66581. The authors also want to thank the comments of participants of the Third World Congress of the Game Theory Society, Evanston 2008 and those of the 20th International Conference on Game Theory, Stony Brook, July 2009. Special thanks go to Sergiu Hart and Josep Peris. The usual disclaimer applies.
} 


\section{INTRODUCTION}

There is an increasing interest in explaining the role of language in human interactions from Economic Theory and Game Theory (see Rubinstein 1996, 2000; Blume 2005; Blume and Board 2008; Segal 2001; Selten and Warglien 2007; Balinski and Laraki 2007a,b, among others). Why is so? Why would such research fields be relevant for linguistic issues? It is well known on the one hand that Economic Theory and Game Theory deal with regularities in human interactions and language is one of the more fundamental ones. On the other hand, both Economic Theory and Game Theory analyze the design of social mechanims and language can be considered a mechanism of communication.

Language is a symbolic system of communication making it possible the inference of meaning. When we nowadays communicate by language, our utterances (signals conveying information within a context) have meaning. In fact, the meaning of a linguistic utterance is not transmitted directly, but is inferred indirectly by the hearer, through pragmatic insights and the social context in which the utterance is received. Furthermore, since linguistic communication became combinatorially richer, then certain markers, alias grammar, started to be used to disambiguate the communicative context ${ }^{1}$.

Language helps human beings to coordinate but coordination takes place many times in different environments and in diverse contexts and here human "natural" languages may experience some difficulties in trying to reduce inefficient outcomes. For instance, empirical analyses have revealed that the use of non-standard or non-native grammatical variants only rarely leads to any communication breakdown, whereas most breakdowns occur due to lexical or phonetic obstacles ${ }^{2}$. To explore the role of languages as coordination devices under communication misunderstandings, we design a symbolic language leading to

\footnotetext{
${ }^{1}$ These considerations lead naturally to queries about the efficiency of language to communicate and learn, inference models of creation of meanings and the role of grammar and categorization in linguistic structures. Several answers have come from Linguistics (Grice, 1975; Gärdenfors, 2000; Azrieli and Lehrer, 2007; Jäger, 2007, etc.), Mathematics and Computer Science (Batali, 1998; Nowak and Krakauer, 1999; Smith, 2003; Kirby, 2002, 2007; Voght, 2005, etc), etc.

${ }^{2}$ In fact, as reported in Reiter and Sripada (2002), linguists have acknowledged that people may associate different meanings with the same word.
} 
coordination. The language that we propose is pragmatic in the sense of Grice (1975). Pragmatics examines the influence of context $^{3}$ on the interpretation of an utterance.

Our context is a Sender-Receiver game where there may be communication misunderstandings ${ }^{4}$. To formally develop this idea, our model specifies to an incomplete information Sender-Receiver game with noisy information transmission. The informed Sender, has to tell the uninformed one, the Receiver, which action to choose. The set of the Sender's meanings is the set of the Receiver's actions. To communicate the Sender encodes her meanings in a set of public signals which are sent to the Receiver. Each signal can, in principle, be anything, for example, letters or merely symbols in an alphabet of salient features which is used to create signals. Signals could be subject to extraneous factors that would distort or interfere with its reception. This unplanned distortion or interference is known as noise ${ }^{5}$. The noise we are concerned with is such that the messages are always received but they can differ from those sent by the Sender, that is, signals may be distorted in the communication process. We model this noise by assuming that each signal can randomly be mapped to the whole set of possible signals ${ }^{6}$ and that the language dictionary is a combination of

\footnotetext{
${ }^{3}$ In Linguistics, a context comprises the speaker, the hearer, the place, the time and so forth. How the hearer views the intentions of the speaker and how the speaker views the presuppositions of the hearer are relevant to the understanding of an utterance.

${ }^{4}$ For instance, though native English speakers may remember 80.000 words, very few of them will use more than 7500 English words in their communication and even in this case communication misunderstandings may appear.

${ }^{5}$ Noise refers to anything introduced into messages that is not included in them by the Sender. Noise may range from mechanical noise, such as the distortion of a voice in the telephone, to any noise generated in human communication such as semantic, psychological or cultural noise. Semantic noise is generated because the ambiguity inherent in all languages and other sign systems; psychological noise occurs when the psychological state of the receiver is such as to produce an unpredictable decoding and cultural noise takes place when the culture or subculture of the audience is so different from that of the sender that messages are understood in ways that the sender might not have anticipated. Such phenomena are pervaisve in many information transmission processes.

${ }^{6}$ In economics there are many situations where "rational" agents have erroneous perceptions, there are signaling models with noise, and, in general, information transmission models with incomplete information such as those of Crawford and Sobel (1982), Lipman and Seppi (1995), Koessler (2001, 2004), among others. In many of these models the noise mainly refers to the strategic uncertainty of the agents about the relevant parameters of the strategic situation under study rather
} 
elements of this set. More precisely, an input sequence is a concatenation of signals. The Sender utters one of these sequences and the Receiver hears an output sequence, which is a probabilistic transformation of the signal string. To isolate the effect of noise from that of strategic uncertainty, it is assumed that the game is one of aligned interests, where the costs of miscoordination are different in distinct states of nature.

The encoding rule or grammar and the decodification rule design a communication device between the Sender and the Receiver. From a given and common knowledge set of public signals and a communication length, a dictionary or "corpus" of sequences is defined, generating the set of standard prototypes which are a one-to-one mapping into the set of Receiver's actions. The structure or grammar specifies that each prototype sequence is positionally arranged and maximally separated from any other sequence. The Receiver has to infer a meaning from each heard sequence or, in other words, to assign an action to each received sequence. Without any noise, the Receiver would accurately infer the action to play from any received prototype. With noise each received sequence belongs to the whole set of possible language sequences and could have been generated from any prototype. Then, the Receiver's criterion is a best-response decoding which partitions the set of possible language sequences into subsets, with a unique action assignment to each of them. In linguistic terms, each of these subset is called the pragmatic variation of a given prototype.

A Language is defined as the pair corpus and pragmatic variations: the way in which the Sender transmits the meanings and that of the Receiver's understandings. We show that our proposed Language is an equilibrium of the noisy communication game: an equilibrium coordination device under interpretation failures. To the best of our knowledge we are the first ones to present an equilibrium approach to the design of pragmatic Languages under general communication

than communication misunderstandings. Blume, Board and Kawamura (2007) examines the possibilities for communication in the Crawford and Sobel's model in a noisy environment. In linguistics, Nowak, Krakauer and Dress (1999) investigate the evolution of communication in the presence of noise: individual may mistake one signal for another. 
misunderstandings. This equilibrium approach is interesting for several reasons. The first one is that it stresses the role of language as a communication mechanism and hence as a coordination device. The proposed pragmatic Language facilitating coordination shares the spirit of Balinski and Laraki (2007)'s work in the Theory of Social Choice. They show that a more "realistic" model in this field, in the sense that messages are grades expressed in a common language, allows preferences to be aggregated. A recent paper on the evolution of language is Demichelis and Weibull (2008), were two parties have a common language and agree on its meaning. They show that such a shared culture -language and honesty code- facilitates coordination on socially efficient equilibrium outcomes in strategic interactions. A different approach is Blume and Board (2008) who take it as given that language is an imperfect technology that leaves messages subject to interpretation. Contrary to us, they investigate the strategic use of interpretable messages.

The second reason deals with the structural regularities of languages. The grammar of our Language ensures the successful transmission ${ }^{7}$ of language itself and universality guarantees the language implementation in very different contexts. Thus, our work stresses that of Nowak and Krakauer (1999) who argue that grammar can be seen as a simplified rule system that reduces the chances of mistakes in implementation and comprehension and it is therefore favored by natural selection in a world where mistakes are possible. Our results also match those of Selten and Warglien (2007), who show in a series of laboratory experiments that in an environment with novelty compositional grammar offer considerable coordination advantages and therefore is more likely to arise.

The third reason is related to communication efficiency. Our pragmatic Language is an efficient inference of meaning model for a not too short communication length, that is, in spite of initial misunderstandings, the Receiver is able to infer with a high probability the Sender's

\footnotetext{
${ }^{7}$ The structure of a language helps to enhance efficiency in communication. In Linguistics Kirby $(2002,2007)$ focuses on the emergence of composition and recursion in languages. In Economics, Rubinstein $(1996,2000)$ is concerned with the structure of binary relations appearing in natural language and Blume (2005) explores the use of structure in languages and how such efficient structures facilitate coordination and learning in repeated coordination games.
} 
meaning. This result guarantees expected payoffs close to those of communication without noise. Furthermore, we characterize the time needed to span the pragmatic variations in order to reduce the chances of misunderstandings and increase expected payoffs. A related paper is Crémer, Garicano and Prat (2007), who characterize efficient technical languages and study their interaction with the scope and structure of organizations.

Finally, the receiver's best reply to any pure sender's strategy induces a partition of the underlying set of public signals around the standard protoypes (as conjectured in linguistic studies, see Gärdenfors 2000). This partition is related to the work on categorization based on prototypes (see Azrieli and Lehrer, 2007, Jäger, 2007 and references herein). The closest result to ours is Jäger (2007), who investigates communication in a partnership signaling game where the set of meanings is equipped with an Euclidean geometrical structure. Under an evolutionary approach, he shows that the sender's strategy partitions the meaning space into quasi-convex categories.

The paper is organized as follows. Section 2 presents the SenderReceiver game and the extended noisy communication game. The existence of a language supporting players' coordination is presented in section 3, where the equilibrium pure strategies for the Sender and the Receiver are constructed. To highlight the main features of our construction we offer some examples in section 4 . In section 5 , the efficiency of our equilibrium for finite communication length is measured and the time needed to approach the full communication payoffs is calculated. Some concluding remarks close the paper.

\section{The MODEL}

2.1. The coordination game. Consider the possibilities of communication between two players, called the Sender $(S)$ and the Receiver $(R)$ in an incomplete information game $\Gamma$ : there is a finite set of feasible states of nature $\Omega=\left\{\omega_{1}, \ldots, \omega_{|\Omega|}\right\}$. Nature chooses first randomly $\omega_{j} \in \Omega$ with probability $q_{j}$ and then the Sender is informed of such state $\omega_{j}$, the Receiver must take some action in some finite action space $A$, and payoffs are realized. The agents' payoffs depend on the Sender's information or type $\omega$ and the Receiver's action $a$. Let 
$u: A \times \Omega \rightarrow R$ be the (common) players' payoff function, i.e., $u\left(a_{t}, \omega_{j}\right)$, $j=0,1, \ldots,|\Omega|-1$. Assume that for each realization $\omega_{j} \in \Omega$, there exists a unique Receiver's action $\widehat{a}_{j} \in A$ with positive payoffs:

$$
u\left(a_{t}, \omega_{j}\right)=\left\{\begin{array}{cl}
M_{j} & \text { if } a_{t}=\widehat{a}_{j} \\
0 & \text { otherwise }
\end{array}\right.
$$

The most $^{8}$ that players can get without any communication is the $\max \left\{q_{j} M_{j}\right\}_{j}$. In Sender-Receiver games, players try to share their private information to achieve coordination and increase their payoffs. They usually communicate using a human or an artificially constructed language. To communicate the Sender encodes the meanings to be transmitted in a set of public signals from the underlying common language. To simplify the model it is assumed that the set of basic signals is the binary alphabet and that the Sender combines elements of this set to communicate. Signals may be distorted in the communication process. This distortion or noise is such that while messages are always received by the Receiver, they may differ from those sent by the Sender. We follow a unifying approach to this noisy information transmission and consider that agents communicate through a discrete noisy channel: a system consisting of input and output alphabets, and a probability transition matrix ${ }^{9}$. Formally, a noisy binary channel $v$ is defined by:

(1) Two sets $X=Y=\{0,1\}$ as the input and the output basic signal sets respectively

(2) A transition probability $p$ : an input signal $s \in X$ is transformed by the channel in an output signal $r \in Y$ with a probability $p(r \mid s)$. Let $\varepsilon_{l}$ be the probability of a mistransmission of the input signal $l$, then since the channel is binary $p(1 \mid 0)=\varepsilon_{0}$ and

\footnotetext{
${ }^{8}$ Suppose that $R$ plays according to the mixed strategy $\alpha=\left(\alpha_{1}, \ldots, \alpha_{|A|}\right)$ that assigns probability $\alpha_{t}$ to action $a_{t}$, then the payoffs obtained by both players are $\sum_{\omega_{j} \in \Omega} q_{j} \alpha M_{j}$. Since this expression is linear on $\alpha$, then the optimal election of the probabilities of $\alpha$ (from the viewpoint of $R$ ) corresponds to a pure strategy (the one corresponding to the vertex $j^{*}$ such that $j^{*}=\arg \max \left\{q_{j} M_{j}\right\}_{j}$ ).

${ }^{9}$ The introduction of noise into a defined channel is well understood in another strand of literature. Such information transmission has been mainly tackled by Information Theory tools. Traditional Information Theory, pioneered by Shannon (1948) abstracts away from equilibrium queries, and focuses on the process of information transmission itself. We will come back to this appoach in subsection 3.1
} 


$$
\begin{aligned}
& p(0 \mid 1)=\varepsilon_{1} \text {. The noisy communication channel is denoted by } \\
& v\left(\varepsilon_{0}, \varepsilon_{1}\right) .
\end{aligned}
$$

We define next what an informative noisy channel is. The key point is that the 'informativeness' of the channel is not related to the probability of properly understanding each basic signal 0 and 1, but instead to the relation between these two probabilities, i.e., to the probability of discriminating between input basic signals once an output basic signal is observed.

Let $s$ be an input signal belonging to some space $X$, and $r$ a realized output signal in space $Y$. Let $p(s)$ be the a priori probability that signal $s$ is delivered through the channel. The channel transforms $s$ into $r$ according to $p(r \mid s)$. From the observed $r$, input signal $s$ is updated by Bayes' rule yielding its posterior conditional probability. Given two output signals $r$ and $\widehat{r}$ and two input signals $s$ and $\widehat{s}$, it is said that $r$ is more favorable than $\widehat{r}$ for $s$, whenever the posterior odds of inputs $s$ and $\widehat{s}$ given the output $r$ are at least as high as those of inputs $s$ and $\widehat{s}$ given the output $\widehat{r}$. Furthermore, the noisy channel $v\left(\varepsilon_{0}, \varepsilon_{1}\right)$ is informative whenever for any realized output signal $r$ and any pair of input signals $s, \widehat{s}: \frac{p(s \mid r)}{p(\widehat{s} \mid r)}=\frac{p(r \mid s) p(s)}{p(r \mid \widehat{s}) p(\widehat{s})} \neq \frac{p(s)}{p(\widehat{s})}$.

Whether the channel is informative and which output signals are more favorable than others are crucial to design the Sender's set of input sequences and the Receiver's decodification procedure. Applying the above concepts to channel $v\left(\varepsilon_{0}, \varepsilon_{1}\right)$, where $s \in\{0,1\}$ and $r \in\{0,1\}$ it is obtained (the proof is given in the Appendix):

Lemma 1. If $\varepsilon_{0}+\varepsilon_{1} \neq 1$, then $v\left(\varepsilon_{0}, \varepsilon_{1}\right)$ is informative. Moreover:

(1) If $\varepsilon_{0}+\varepsilon_{1}<1$, then for input signal 0 , output signal 0 is more favorable than output signal 1, and for input signal 1, output signal 1 is more favorable than output signal 0.

(2) If $\varepsilon_{0}+\varepsilon_{1}>1$, then for input signal 1 output signal 0 is more favorable than output signal 1 , and for input signal 0 , output signal 1 is more favorable than output signal 0.

Noisy channels are only characterized by their levels of aggregate noise. Firstly, if channel $v\left(\varepsilon_{0}, \varepsilon_{1}\right)$ is informative with low levels of aggregate noise $\left(\varepsilon_{0}+\varepsilon_{1}<1\right)$, then the matching between the output and the input signal will yield a more accurate posterior odds. For 
symmetric channels, i.e. $\varepsilon_{0}=\varepsilon_{1}=\varepsilon$, this condition implies that the misunderstandings are not too high, i.e. $\varepsilon<\frac{1}{2}$. Nevertheless, $\varepsilon_{0}=0.2$ and $\varepsilon_{1}=0.7$ in asymmetric channels also fulfil the condition. Secondly, when channel $v\left(\varepsilon_{0}, \varepsilon_{1}\right)$ is informative with high levels of aggregate noise $\left(\varepsilon_{0}+\varepsilon_{1}>1\right)$, then the unmatching between the output and the input signal will yield now a more accurate posterior odds. Finally, when $\varepsilon_{0}+\varepsilon_{1}=1$, there is no way to discriminate between input signals once an output signal is observed and the channel is not-informative. This happens when $\varepsilon=\frac{1}{2}$ in symmetric channels, or, for instance, when $\varepsilon_{0}=0.1$ and $\varepsilon_{1}=0.9$ in asymmetric ones.

Communication goes on for $n$ periods. It is also assumed that the channel is memoryless, i.e., the probability distribution of the output depends only on the input at that time and is conditionally independent of previous channel inputs or outputs. Then, given a communication length $n$, the Sender utters to the channel an input sequence, $x$ $\in X^{n}=\{0,1\}^{n}$, which is a concatenation of basic binary signals and the Receiver hears an output sequence, $y \in Y^{n}=\{0,1\}^{n}$, which is an independent probabilistic transformation of the signal string. Thus $\Gamma$ is extended by a pre-play phase of communication where the Sender uses $n$ times the channel $v\left(\varepsilon_{0}, \varepsilon_{1}\right)$. Let $\Gamma_{v}^{n}$ denote this extended communication game, where after the communication stage the uninformed player $R$ chooses an action from her action space (infers a meaning from $y$ and the game context) upon observing output sequence $y$ and then payoffs are realized.

Since the Sender encodes her meanings in a set of public signals, her strategy in $\Gamma_{v}^{n}$ is a rule suggesting the message to be sent at each $\omega_{j}$ : a sequence $\sigma_{j}^{S} \in X^{n}$ sent by $S$ given that the true state of nature is $\omega_{j}$. Each sequence $\sigma_{j}^{S}$ is called a standard prototype. The set of standard prototypes $\left\{\sigma_{j}^{S}\right\}_{j}$ is the corpus.

A strategy of $R$ is a $2^{n}$-tuple $\left\{\sigma_{y}^{R}\right\}_{y}$, where $\sigma_{y}^{R}$ specifies an action choice as a response to the realized output sequence $y \in\{0,1\}^{n}$. The action associated by $R$ to each sequence, jointly with the context, is the meaning decodification. Then, a Receiver's strategy is the inference of a meaning for any sequence in the language, even for those not included in the corpus. An univocal construction of meanings partitions the set of output sequences $Y^{n}$ into $|\Omega|$ subsets, each of them bringing together 
all the sequences whose meaning is the choice of action $\widehat{a}_{j}, j=1, \ldots$, $|\Omega|$. Each subset of this partition is the pragmatic variation associated to a particular standard prototype.

A Language is the pair corpus and pragmatic variations: the Sender's meaning transmission and the Receiver's understandings. An equilibrium Language is that for which the players' strategies are a best response to each other.

Expected payoffs in $\Gamma_{v}^{n}$ are defined in the usual way. Let the tuple of the Sender's expected payoffs be denoted by: $\left\{\pi_{j}^{S}\right\}_{j}=\left\{\pi_{j}^{S}\left(\sigma_{j}^{S},\left\{\sigma_{y}^{R}\right\}_{y}\right)\right\}_{j}$, where for each $\omega_{j}$,

$$
\pi_{j}^{S}=\pi_{j}^{S}\left(\sigma_{j}^{S},\left\{\sigma_{y}^{R}\right\}_{y}\right)=\sum_{y \in Y^{n}} p\left(y \mid \sigma_{j}^{S}\right) u\left(\sigma_{y}^{R}, \omega_{j}\right)
$$

and where $p\left(y \mid \sigma_{j}^{S}\right)$ is the Sender's probability about the realization of the output sequence $y \in\{0,1\}^{n}$ conditional on having sent sequence $\sigma_{j}^{S}$ in state $\omega_{j}$.

Let the tuple of the Receiver's expected payoffs be $\left\{\pi_{y}^{R}\right\}_{y}=\left\{\pi_{y}^{R}\left(\left\{\sigma_{j}^{S}\right\}_{j}, \sigma_{y}^{R}\right)\right\}_{y}$, where for each output sequence $y \in\{0,1\}^{n}$,

$$
\pi_{y}^{R}=\pi_{y}^{R}\left(\left\{\sigma_{j}^{S}\right\}_{j}, \sigma_{y}^{R}\right)=\sum_{j=1}^{|\Omega|} p\left(\sigma_{j}^{S} \mid y\right) u\left(\sigma_{y}^{R}, \omega_{j}\right)
$$

and where $p\left(\sigma_{j}^{S} \mid y\right)$ is the Receiver's probability about input sequence $\sigma_{j}^{S}$ in state $\omega_{j}$ conditional on having received the output sequence $y$.

A pure strategy Bayesian Nash equilibrium of the communication game is a pair of tuples $\left(\left\{\widehat{\sigma}_{j}^{S}\right\}_{j},\left\{\widehat{\sigma}_{y}^{R}\right\}_{y}\right)$, i.e., a Sender's corpus and a Receiver's sets of pragmatic variations, and a set of probabilities $\left\{p\left(\sigma_{j}^{S} \mid y\right)\right\}_{j}$ for the Receiver such that for each $\omega_{j}$, and for any other strategy $\widetilde{\sigma}_{j}^{S}$ of the Sender,

$$
\widehat{\pi}_{j}^{S}=\pi_{j}^{S}\left(\widehat{\sigma}_{j}^{S},\left\{\widehat{\sigma}_{y}^{R}\right\}_{y}\right) \geq \pi_{j}^{S}\left(\widetilde{\sigma}_{j}^{S},\left\{\widehat{\sigma}_{y}^{R}\right\}_{y}\right),
$$

and for each $y \in\{0,1\}^{n}$ and for any other Receiver's strategy $\widetilde{\sigma}_{y}^{R}$,

$$
\widehat{\pi}_{y}^{R}=\pi_{y}^{R}\left(\left\{\widehat{\sigma}_{j}^{S}\right\}_{j}, \widehat{\sigma}_{y}^{R}\right) \geq \pi_{y}^{R}\left(\left\{\widehat{\sigma}_{j}^{S}\right\}_{j}, \widetilde{\sigma}_{y}^{R}\right),
$$

where by Bayes rule each $p\left(\sigma_{j}^{S} \mid y\right)$ is given by: $p\left(\sigma_{j}^{S} \mid y\right)=\frac{p\left(y \mid \sigma_{j}^{S}\right) p\left(\sigma_{j}^{S}\right)}{p(y)}$.

The ex-ante expected payoffs of this communication game are given by

$$
\Pi^{S}\left(\left\{\sigma_{j}^{S}\right\}_{j},\left\{\sigma_{y}^{R}\right\}_{y}\right)=\sum_{j=1}^{|\Omega|} q_{j} \pi_{j}^{S}\left(\sigma_{j}^{S},\left\{\sigma_{y}^{R}\right\}_{y}\right)=\sum_{j=1}^{|\Omega|} q_{j} \sum_{y \in Y^{n}} p\left(y \mid \sigma_{j}^{S}\right) u\left(\sigma_{y}^{R}, \omega_{j}\right)
$$


for the Sender an those of the Receiver are defined by,

$$
\Pi^{R}\left(\left\{\sigma_{j}^{S}\right\}_{j},\left\{\sigma_{y}^{R}\right\}_{y}\right)=\sum_{y \in Y^{n}} p(y) \pi_{y}^{R}\left(\left\{\sigma_{j}^{S}\right\}_{j}, \sigma_{y}^{R}\right)=\sum_{y \in Y^{n}} p(y) \sum_{j=1}^{|\Omega|} p\left(\sigma_{j}^{S} \mid y\right) u\left(\sigma_{y}^{R}, \omega_{j}\right)
$$

Notice that $\Pi^{S}\left(\left\{\sigma_{j}^{S}\right\}_{j},\left\{\sigma_{y}^{R}\right\}_{y}\right)=\Pi^{R}\left(\left\{\sigma_{j}^{S}\right\}_{j},\left\{\sigma_{y}^{R}\right\}_{y}\right)$, since $\Gamma$ is symmetric and $\sum_{j=1}^{|\Omega|} \sum_{y \in Y^{n}} q_{j} p\left(y \mid \sigma_{j}^{S}\right)=\sum_{y \in Y^{n}} \sum_{j=1}^{|\Omega|} p(y) p\left(\sigma_{j}^{S} \mid y\right)$. Denote this common ex-ante expected payoffs by $\Pi_{v}$.

In order to distinguish among sequences, a distance function among them has to be defined. A natural and intuitive function is the Hamming distance. Formally, consider two $n$-dimensional sequence $x=$ $\left(x^{1}, \ldots, x^{n}\right)$ and $y=\left(y^{1}, \ldots, y^{n}\right)$. Let $I$ stands for the indicator function. The Hamming distance between two sequences $x, y$, denoted $h(x, y)$, is defined as $h(x, y)=\sum_{t=1}^{n} I_{x^{t} \neq y^{t}}$.

Suppose that $n=m|\Omega|$. For $1 \leq l \leq|\Omega|$, the $l$-block of length $m$ of $x=\left(x^{1}, \ldots, x^{n}\right)$ is the subsequence $\left(x^{(l-1) m+1}, \ldots, x^{l m}\right)$. The Hamming distance in the $l$-th block between $x$ and $y$, denoted by $h_{l}(x, y)$ is equal to the Hamming distance between the $l$-th block of $x$ and $l$-th block of $y$. Formally,

$h_{l}(x, y)=h\left(\left(x^{(l-1) m+1}, \ldots, x^{l m}\right),\left(y^{(l-1) m+1}, \ldots, y^{l m}\right)\right)=\sum_{t=1}^{m} I_{x^{(l-1) m+t} \neq y^{(l-1) m+t}}$

By additivity, the Hamming distance between $x$ and $y$ coincides with the sum of the Hamming distances of the $|\Omega|$-blocks between $x$ and $y$ : $h(x, y)=\sum_{l=1}^{|\Omega|} h_{l}(x, y)$.

\section{Pure Equilibrium Strategies under informative Noisy CHANNELS.}

Our main finding shows the existence of an equilibrium pragmatic Language, with a universal structure or grammar. In fact, we show how to construct such a Language. Language is pragmatic in the sense that the Receiver' decoding rule depends on the context, i.e, on the payoffs and on the initial probability distribution of the states of nature of $\Gamma$. Language has a universal grammar because the Sender's corpus does not depend on such specific parameters of $\Gamma$ and can then be applied to any Sender-Receiver game. Both rules are a best response to each other, generating an equilibrium Language.

In the noisy communication game $\Gamma_{v}^{n}$, the cardinality of the set of communication sequences exceeds that of the set of states of nature. 
Then, given the set of basic signals $\{0,1\}$ and $n$, a dictionary or "corpus" of sequences from $\{0,1\}^{n}$ is designed by selecting $|\Omega|$ of them, one for each state of nature. Each selected sequence is a standard prototype, $\left\{\sigma_{j}^{S}\right\}_{j \in\{1, \ldots,|\Omega|\}}$. Standard prototypes are constructed by dividing any input sequence $\left(x_{j}^{1}, \ldots, x_{j}^{n}\right)$ in $|\Omega|$ blocks of length $m$ in such a way that all blocks but the $j$-th consist of repetitions of signal 1 and the $j$-th block is composed of $m$ repetitions of signal 0 . For each realized state of nature, the Sender utters to the channel a standard prototype. A Sender's pure strategy is then the standard prototype to be sent at each $\omega_{j}$.

For each prototype, the noise induces any output sequence in $Y^{n}=$ $\{0,1\}^{n}$, say $y$. Once $y$ is observed, the Receiver chooses his best response. His choice is based on the following meaning inference procedure: given the noisy information transmission, he partitions the set of all possible received sequences $\{0,1\}^{n}$ in a collection of subsets which are called "the pragmatic variation classes", denoted by $\left\{\sigma_{y}^{R}\right\}_{y}$. Each pragmatic variation class is associated to a particular standard prototype and hence to a particular action. Therefore, the Receiver will play the action dictated by the pragmatic variation including output sequence $y$. Since the Receiver maps the observed output sequence into prototypes, his best reply to any pure Sender's strategy induces a kind of "categorization" 10 of the output space, $Y^{n}=\{0,1\}^{n}$, around the standard protoypes. Thus, at equilibrium the output message space is partitioned in a finite number of sets.

Given that the Sender knows the Receiver's partition of the output space, her best response is truth-telling. Specifically, the Sender has no incentive to lie uttering an input sequence different from the corresponding standart prototype. This is so because the probability mass of the pragmatic variation class associated to each standard prototype (and hence to each state of nature) only decreases by lying. Therefore, the main result states that the transmission of the corresponding standard prototype (for a realized output sequence) by the Sender, and the choice of the action suggested by the classes of pragmatic variations by the Receiver are a pure strategy Bayesian Nash equilibrium of $\Gamma_{v}^{n}$.

\footnotetext{
${ }^{10}$ Categorization is the way by which a set of entities, identified with some finite dimensional Euclidean space, is partitioned into a finite number of categories. Categories are sets of entities to which we react in an identical or similar way.
} 
Firstly, we present the Theorem, proven in the Appendix, and then we show how to construct such strategies.

Theorem 1. There exists an $n_{0} \in \mathbb{N}$ such that for all $n \geq n_{0}$ the pair of tuples $\left(\left\{\widehat{\sigma}_{j}^{S}\right\}_{j},\left\{\widehat{\sigma}_{y}^{R}\right\}_{y}\right)$ and the set of probabilities $\left\{p\left(\sigma_{j}^{S} \mid y\right)\right\}_{j}$ is a pure strategy Bayesian Nash equilibrium of $\Gamma_{v}^{n}$.

The partition of the output space in the pragmatic variations of the standard prototypes is related to the work of Azrieli and Lehrer(2007), who suggest a categorization model based on prototypes. Our result is also related to Jäger (2007), who analyzes the class of Sender-Receiver games, where the cardinality of the set of meanings exceeds the size of the set of signals by several orders of magnitude. He shows that under the replicator dynamics, a strict equilibrium set is such that for each Receiver's pure strategy, the Sender's best reply is a categorization of the meaning space.

On the contrary, in our noisy communication game, the Receiver's best reply to any pure Sender's strategy induces a categorization of the output message space around the standard protoypes. This partition is due to an inference process rather than to an evolutionary dynamics. Thus, noisy communication processes induce pragmatic categorizations of the message space. Unlike Jäger, the players' utility function reflects different payoffs under different standard prototypes, this meaning that it is the weighted (by expected payoffs) distance what matters for categorization. Our categorization takes then into account not only the pure similarity or distance to the standard prototypes but also the expected payoffs of game $\Gamma$.

In the sequel, we offer the construction of the pure equilibrium strategies for the Sender and the Receiver, i.e. $\left\{\widehat{\sigma}_{j}^{S}\right\}_{j}$ and $\left\{\widehat{\sigma}_{y}^{R}\right\}_{y}$, respectively, in $\Gamma_{v}^{n}$ when the noisy channel $v\left(\varepsilon_{0}, \varepsilon_{1}\right)$ is informative with low levels of aggregate noise, i.e., $\varepsilon_{0}+\varepsilon_{1}<1$. The remaining case is similar and we omit it.

\subsection{The corpus and the pragmatic variations of the standard}

prototypes. One is tempted to look at Information Theory to design the players' coding and decoding strategies in our noisy communication game $\Gamma_{v}^{n}$. More specifically, Coding Theory is concerned with the design of practical encoding and decoding systems to achieve reliable 
communication over a noisy channel. The general idea is that the encoding system introduces systematic redundancy into the transmitted message, while the decoding system uses this known redundancy to deduce from the received message both the original source vector and the noise introduced by the channel ${ }^{11}$. The basic Theorem of Information Theory is then the achievability of the channel capacity by a communication protocol (based on encoding and decoding rules) under the implicit assumption that the two communicating agents commit exante to following a particular encoding and decoding strategies before the communication stage.

In game theoretical analysis players are required to take actions when they are called upon to do so, therefore given an encoding rule and an output message, the Receiver's equilibrium conditions summarize the choice of the action corresponding to that state of nature for which his expected payoffs are higher. Thus, unlike Information Theory, the role of a decoding rule in our problem is not that of recovering a string potentially perturbed by the noise channel but instead that of inferring at equilibrium which of the actual $|\Omega|$ valid messages was actually sent through the channel. Since we are interested in encoding systems supporting equilibria, there is only one feasible decoding rule which is given by the 'best response' decoding rule. Obviously, not all coding and decoding rules $^{12}$ from Information Theory can generate the conditions to satisfy the Nash equilibrium requirements. This is so, even when players' strategies come from a well-established theory guaranteeing a good rate of information transmission (see, Hernandez, Urbano and Vila, 2009). For this reason the application of standard encoding systems, which are more efficient in terms of transmission rates may not be too appropriated when designing simple and universal encoding systems supporting equilibria.

\footnotetext{
${ }^{11}$ There are mainly two different families of encoding rules for binary noisy channels: repetition codes and linear block codes (where the most known are the Hamming codes).

${ }^{12}$ Decoding rules associated to standard code systems as for instance either the Hamming code $(7,4)$ or random codes may not be, in general, best responses to the received string.
} 
The next step is to design the encoding ${ }^{13}$ rule or corpus of the standard prototypes which is a best response to the Receiver's decoding. A coding rule for our problem is a rule assigning a string of $n$ symbols in $\{0,1\}$ to each state of nature. We will use a variation of a repetition ${ }^{14}$ code: the block coding rule. A general characterization of the Receiver's best response decoding rule for any feasible Sender's corpus would depend of the game parameters, the noise and the encoding parameters (the interested reader may consult the authors' web pages) ${ }^{15}$. To leave apart the encoding parameters, and focus on the game theoretical aspects of the problem, we construct an easy and universal encoding rule allowing a simple characterization (for instance, in terms of the Hamming distance) of the best response decoding rule, only depending on both the game and the noise parameters of any Sender-Receiver game with noisy communication. As it will become clear in the sequel the block coding rule is independent of the game payoffs and of the initial probabilities of the sates of nature.

3.1.1. The Corpus: Block Coding. The corpus is constructed from the set of basic signals $\{0,1\}$ and the communication length. For a given length $n$, the set of possible utterances is then $\{0,1\}^{n}$. Since each state $\omega_{j}$ is associated with a receiver's optimal action $\widehat{a_{j}}$, then, the corpus consists of the $|\Omega|$ sequences in $\{0,1\}^{n}$ given by $\left\{\widehat{\sigma}_{1}^{S}, . ., \widehat{\sigma}_{|\Omega|}^{S}\right\}$ and each of the $|\Omega|$ sequences $\widehat{\sigma}_{j}^{S}$ is the standard prototype encoding the meaning "take the action $\widehat{a_{j}}$ ". The Sender's pure strategy assigns to each state $\omega_{j}$ a tuple $\widehat{\sigma}_{j}^{S}=\left(x_{j}^{1}, \ldots, x_{j}^{n}\right)$. Assume that the number of states of nature $|\Omega|$ is a multiple ${ }^{16}$ of $n$, i.e., there exists an integer $m$ such that $n=m|\Omega|$.

Since many sequences in $\{0,1\}^{n}$ are possible, some grammar is needed to isolate structural regularities. In particular, our language grammar

\footnotetext{
${ }^{13}$ The framework in our paper is quite different from that of Coding Theory. In particular, the number of states of nature (the cardinality of $\Omega$ ), from which one has to be transmitted, is fixed and usually small.

${ }^{14}$ Repetition codes generate redundancy by the repetition of every bit of the message a prearranged number of times. This family of codes can achieve arbitrarily small probability of error only by decreasing the rate of transmission. However, they are useful for many practical purposes as, for instance, when universality is a desiderable property.

${ }^{15}$ Either http://www.uv.es/penelope/ or http://www.uv.es/urbano/

${ }^{16}$ If $n$ is not a multiple of $m$, one may consider $m$ as the integer part of $\frac{n}{[\Omega]}$. The remaining elements would be considered without meaning.
} 
is based on a block structure which allows us to construct a corpus as follows: each $\widehat{\sigma}_{j}^{S} \in X^{n}=\left(x_{j}^{1}, \ldots, x_{j}^{n}\right)$ where the element

$$
x_{j}^{i}=\left\{\begin{array}{cc}
0 & \text { if }(j-1) m-1 \leq i \leq j m \\
1 & \text { otherwise }
\end{array}\right.
$$

In other words, input sequence $\left(x_{j}^{1}, \ldots, x_{j}^{n}\right)$ is divided into $|\Omega|$ blocks of length $m$ in such a way that all blocks but the $j$-th consist of repetitions of signal 1 and the $j$-th block is the $m$-repetition of signal 0 . Thus, the structure or grammar specifies that each prototype sequence is positionally arranged, that is, in blocks. A first property of this grammar is that prototypes have the maximal separation among them. Furthermore, the block structure permits the block by block comparison among any sequence $y$ and all the prototypes. Thus, the relevant information when comparing output sequence $y$ to prototype $\widehat{\sigma}_{l}^{S}$ is only contained in the corresponding block $l$. Moreover, all the remaining prototypes give the same block $l$-information with respect to $\sigma_{l}^{S}$. The following lemma formalizes this property, where the block Hamming distance between sequences is the distance measure.

Lemma 2. For all $k, k^{\prime} \in\{1,2, \ldots,|\Omega|\}$, we have that

1. $h_{l}\left(\widehat{\sigma}_{k}^{S}, y\right)=h_{l}\left(\widehat{\sigma}_{k^{\prime}}^{S}, y\right)$ if $k \neq l \neq k^{\prime}$

2. $h_{k}\left(\widehat{\sigma}_{k}^{S}, y\right)+h_{k}\left(\widehat{\sigma}_{k^{\prime}}^{S}, y\right)=m$ if $k \neq k^{\prime}$

The players' strategies can be understood as a communication protocol. One of the most desired properties in communication protocol design is universality. The corpus satisfies this property since it does not depend on the specific parameters of $\Gamma$, that is, on the payoffs and the initial probability distribution of the states of nature.

3.1.2. The Receiver's best response: The pragmatic variations of the standard prototypes. The Receiver has to take the action in $\Gamma$, after hearing an output sequence $y$, which maximizes his expected payoffs. Equivalently, for each $y$ he chooses the action $\widehat{a}_{l}(y)$ such that

$$
\sum_{j=1}^{|\Omega|} p\left(\widehat{\sigma}_{j}^{S} \mid y\right) u\left(\widehat{a}_{l} \mid \omega_{j}\right) \geq \sum_{j=1}^{|\Omega|} p\left(\widehat{\sigma}_{j}^{S} \mid y\right) u\left(a_{k} \mid \omega_{j}\right),
$$

for any other $k \neq l$ which, given both the linearity of the Receiver's payoff functions in probabilities $\left\{p\left(\sigma_{l}^{S} \mid y\right)\right\}_{l}, l=1, \ldots,|\Omega|$, and the 
matrix payoffs is equal to $p\left(\widehat{\sigma}_{l}^{S} \mid y\right) M_{l} \geq p\left(\widehat{\sigma}_{k}^{S} \mid y\right) M_{k}$, or

$$
\frac{p\left(\widehat{\sigma}_{l}^{S} \mid y\right)}{p\left(\widehat{\sigma}_{k}^{S} \mid y\right)} \geq \frac{M_{k}}{M_{l}}
$$

The next proposition states that given a noisy communication channel $v=\left\{\varepsilon_{0}, \varepsilon_{1}\right\}$ and an observed output sequence $y$, the likelihood ratio of any two standard prototypes, say $\widehat{\sigma}_{j}^{S}$ and $\widehat{\sigma}_{k}^{S}$, can be written in terms of their block hamming distance with respect to output signal $y$ and of the channel noise parameters. In the Appendix some easy but cumbersome calculations show that.

Proposition 1. For all $k, l=1, \ldots,|\Omega|, k \neq l$ and for all $y \in Y$,

$$
\frac{p\left(\widehat{\sigma}_{l}^{S} \mid y\right)}{p\left(\widehat{\sigma}_{k}^{S} \mid y\right)}=\frac{q_{l}}{q_{k}}\left(\frac{\varepsilon_{0}}{1-\varepsilon_{0}} \frac{\varepsilon_{1}}{1-\varepsilon_{1}}\right)^{h_{l}\left(\widehat{\sigma}_{l}^{S}, y\right)+h_{k}\left(\widehat{\sigma}_{l}^{S}, y\right)-m}
$$

Substitution of (2) in (1) gives an expression which helps the Receiver to assign each sequence $y$ to one of the standard prototypes and hence to a specific action. This assignment is based on both the number of different elements (errors) that each two standard prototype sequences $\widehat{\sigma}_{l}^{S}$ and $\widehat{\sigma}_{k}^{S}$ may have with respect to the observed output sequence $y$ and on the ratio of their associated expected payoffs $\frac{M_{k} q_{k}}{M_{l} q_{l}}$. More precisely, the Receiver's pure equilibrium strategy generates first a partition of output set $Y^{n}$ around the standard prototypes based on both the prototype likelihood ratio and their relative expected payoffs.

To proceed with the construction of the message space partition, compute first parameters $\left\{C_{l k}\right\}_{l \neq k}$, for each $l, k \in\{1, \ldots,|\Omega|\}$, as the integer approximation of the number coming from the expression:

$$
\frac{\ln \frac{M_{k} q_{k}}{M_{l} q_{l}}}{\ln \frac{\varepsilon_{0}}{1-\varepsilon_{0}} \frac{\varepsilon_{1}}{1-\varepsilon_{1}}}+m
$$

Parameters $\left\{C_{l k}\right\}_{l \neq k}$ are the "vicinity bounds"; there are $|\Omega| \times(|\Omega|-$ 1) of them and can be arranged in the following way,

$$
\begin{array}{lllll}
Y_{1} & Y_{2} & Y_{3} & Y_{l} & Y_{|\Omega|}
\end{array}
$$




$$
\left(\begin{array}{ccccccc}
* & C_{21} & C_{31} & \cdots & C_{l 1} & \cdots & C_{|\Omega| 1} \\
C_{12} & * & C_{32} & \cdots & C_{l 2} & \cdots & C_{|\Omega| 2} \\
C_{13} & C_{23} & * & \cdots & C_{l 3} & \cdots & C_{|\Omega| 3} \\
\cdots & \cdots & \cdots & \cdots & \cdots & \cdots & \cdots \\
C_{1 l} & C_{2 l} & C_{3 l} & \cdots & * & \cdots & C_{|\Omega| l} \\
\cdots & \cdots & \cdots & \cdots & \cdots & \cdots & \cdots \\
C_{1|\Omega|} & C_{2|\Omega|} & C_{3|\Omega|} & \cdots & C_{l|\Omega|} & \cdots & *
\end{array}\right)
$$

where each column gives the constraints defining subsets of $Y^{n}$ and with typical element $C_{l k}$. Each $C_{l k}$ is an upper bound on the distance between blocks $l$ and $k$ in $y$ and the corresponding ones in $\widehat{\sigma}_{l}^{S}$. Thus, parameter $C_{l k}$ is the maximum number of permitted mistakes to infer that output sequence $y$ comes from $\widehat{\sigma}_{l}^{S}$ instead of coming from $\widehat{\sigma}_{k}^{S}$. For a given noisy channel $v\left(\varepsilon_{0}, \varepsilon_{1}\right)$ and communication length $n$, expression (3) measures the relative expected payoff loss from taking one action instead of the other. Therefore the minimum over the $k$ 's of vicinity bounds $\left\{C_{l k}\right\}_{k}, k \neq l$, is the maximum of such relative expected payoff losses.

To precisely specify each subset $Y_{l}$, we divide the set of states of nature in two subsets:

(1) One containing those states $k$, different from states $l$ in expected payoffs, that is $q_{l} M_{l} \neq q_{k} M_{k}$. Let $\tilde{\Omega}_{l}=\left\{k \in\{1, \ldots,|\Omega|\}\right.$ such that $q_{l} M_{l} \neq$ $\left.q_{k} M_{k}\right\}$ be such a $\operatorname{set}^{17}$.

(2) Another one with the remaining states where their expected payoffs coincide with those of state $l$. In this (symmetric) later case, a rule to break ties is needed. Our rule is the same for every pragmatic variation, coincides with the length of the block and is independent of both the noise and the expected payoffs.

We are now ready to define subsets $Y_{l}$ of the partition. Each subset is determined by vicinity bounds on the number of permitted block hamming distances (errors) ${ }^{18}$ between a standard prototype $\widehat{\sigma}_{l}^{S}$ and

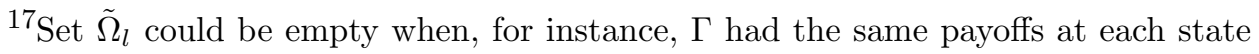
and the priors were uniformly distributed.

${ }^{18}$ Recall that we are considering informative channels with low levels of aggregate noise, $\varepsilon_{0}+\varepsilon_{1}<1$. Here, the matching between the output and the input signal yields a more accurate posterior odds.
} 
the realized output sequence $y$.

$$
\begin{aligned}
Y_{l}=\left\{y \in Y^{n} \mid \quad\right. & h_{l}\left(\widehat{\sigma}_{l}^{S}, y\right)+h_{k}\left(\widehat{\sigma}_{l}^{S}, y\right) \leq C_{l k}, \text { for all } k \in \tilde{\Omega}_{l} \\
& h_{l}\left(\widehat{\sigma}_{l}^{S}, y\right)+h_{k^{\prime}}\left(\widehat{\sigma}_{l}^{S}, y\right) \leq C_{l k^{\prime}}, \text { for all } k^{\prime} \epsilon \notin \tilde{\Omega}_{l}, k^{\prime}<l \\
& \left.h_{l}\left(\widehat{\sigma}_{l}^{S}, y\right)+h_{k^{\prime}}\left(\widehat{\sigma}_{l}^{S}, y\right)<C_{l k^{\prime}}, \text { for all } k^{\prime} \notin \tilde{\Omega}_{l}, k^{\prime}>l\right\}
\end{aligned}
$$

Finally, for each realized $y$, the Receiver's pure equilibrium strategy is

$$
\widehat{\sigma}_{y}^{R}=\widehat{a}_{l} \Leftrightarrow y \in Y_{l}
$$

It is worth remarking:

1. Given the above description of $Y_{l}$, and that either $C_{l k}+C_{k l}=$ $2 m-1$ whenever $q_{l} M_{l} \neq q_{k} M_{k}$ or $C_{l k}+C_{k l}=2 m$ whenever $q_{l} M_{l}=$ $q_{k} M_{k}$, sets $Y_{l}, l=\{1, \ldots,|\Omega|\}$, are a true partition of $Y^{n}$ (see the Appendix). Therefore, the Receiver's best reply to any pure Sender's strategy induces a categorization of the output message space $Y^{n}=$ $\{0,1\}^{n}$ around the standard protoypes.

2. The necessary condition to have a non-empty $Y_{l}$ is that the sign of all the $C_{l k}$ 's is non-negative. As the expression $\ln \left(\frac{\varepsilon_{0}}{1-\varepsilon_{0}} \frac{\varepsilon_{1}}{1-\varepsilon_{1}}\right)$ is negative, whenever both $q_{k} M_{k}>q_{l} M_{l}$ and the communication length $n$ is very short, i.e., $m=\frac{n}{|\Omega|}$ is not big enough, then the associated $C_{l k}$ may be negative. This will give rise to not playing at all the corresponding action $\widehat{a}_{l}$ and an efficiency loss. In section 5, we will show how to cope with this case and improve efficiency by designing an auxiliary truncated game.

3. Finally, let us specify the size of the standard prototype block, $m$, in parameters $\left\{C_{l k}\right\}_{l \neq k}$ by denoting each of them by $C_{l k}^{m}$. If the communication length increases up to $n+|\Omega|$, then the new block length will be $m^{\prime}=\frac{n+|\Omega|}{|\Omega|}=m+1, C_{l k}^{m^{\prime}}=\frac{\ln \frac{M_{k} q_{k}}{M_{l} q_{l}}}{\ln \frac{\varepsilon_{0}}{1-\varepsilon_{0}} \frac{\varepsilon_{1}}{1-\varepsilon_{1}}}+m+1$, and thus $C_{l k}^{m^{\prime}}=$ $C_{l k}^{m}+1$. Therefore, parameters, $C_{l k}^{m}$ grows by a unit whenever the block length $m=\frac{n}{|\Omega|}$ increases by one. This means that as $n$ increases, the vicinity bounds and hence the number of sequences belonging to each $Y_{l}, l=\{1, \ldots,|\Omega|\}$, increase as well. This fact is useful to characterize the time needed to span the pragmatic variations in order to approach any targeted expected payoffs. 
The assignment process of the specific sequences belonging to each $Y_{l}$ is a little cumbersome but the following examples nicely illustrate the whole construction.

\section{ExAmples}

Example 1. Consider the following Sender-Receiver game $\Gamma$ with three states of nature where nature chooses $\omega_{j}, j=1,2,3$ according to law $q=\left(q_{1}, q_{2}, q_{3}\right)=(0.5,0.25,0.25)$. The set of actions for player $R$ is $A=\left\{a_{1}, a_{2}, a_{3}\right\}$, and payoffs for the three states of nature are $M_{1}=1$, $M_{2}=7$ and $M_{3}=43$, or in matrix form:

$$
\left.\begin{array}{llll} 
& a_{1} & a_{2} & a_{3} \\
\omega_{1} & (1,1) & (0,0) & (0,0) \\
\omega_{2} & (0,0) & (7,7) & (0,0) \\
\omega_{3} & (0,0) & (0,0) & (43,43)
\end{array}\right)
$$

Suppose that the players communicate $n=6$ times through the noisy channel $v\left(\varepsilon_{0}, \varepsilon_{1}\right)$ with associated transition probabilities $p(1 \mid 0)=$ $\varepsilon_{0}=0.1$ and $p(0 \mid 1)=\varepsilon_{1}=0.6$. Thus, the communication channel is $v=\{0.1,0.6\}$ and $\Gamma_{v}^{6}$ is the associated extended communication game.

For each state $\omega_{j}, j=1,2,3$, the Sender divides the standard prototype sequences $\left(x_{j}^{1}, \ldots, x_{j}^{6}\right) \in X^{6}$, in 3 blocks of length $m=\frac{6}{3}=2$, where the $j$-th block, consists of two consecutive 0 's and the other blocks of two consecutive 1's. Thus, the corpus consists of the Sender's 3-tuple of standard prototypes: $\widehat{\sigma}_{1}^{S}=001111, \widehat{\sigma}_{2}^{S}=110011$ and $\widehat{\sigma}_{3}^{S}=$ 111100.

To begin with the partition of output message space $Y=\{0,1\}^{6}$, the Receiver considers the matrix of elements $C_{l k}=\frac{\ln \frac{M_{k} q_{k}}{M_{l} q_{l}}}{\ln \frac{\varepsilon_{1}}{1-\varepsilon_{0}} \frac{\varepsilon_{1}}{1-\varepsilon_{1}}}+m$,

$$
\begin{gathered}
C_{1 k} \\
k=1 \\
k=2 \\
k=3
\end{gathered} \quad\left(\begin{array}{ccc}
* & C_{2 k} & C_{3 k} \\
1 & * & 3 \\
0 & 0 & *
\end{array}\right)
$$

Next, given that for all $j=1,2,3, q_{j} M_{j} \neq q_{i} M_{i}, i \neq j$, output set $Y=$ $\{0,1\}^{6}$ is partitioned by the Receiver in subsets $Y_{l}=\left\{y \mid h_{l}\left(\widehat{\sigma}_{l}^{S}, y\right)\right)+$ $\left.\left.h_{k}\left(\widehat{\sigma}_{l}^{S}, y\right)\right) \leq C_{l k}, \forall k, l=1,2,3, k \neq l\right\}$, where $\left\{Y_{1}, Y_{2}, Y_{3}\right\}$ are defined 
by the above matrix as follows:

$$
\begin{aligned}
& Y_{1}=\left\{y \in\{0,1\}^{6} \quad \begin{array}{l}
\mid h_{1}(001111, y)+h_{2}(001111, y) \leq 1=C_{12} \\
\mid h_{1}(001111, y)+h_{3}(001111, y)=0=C_{13}
\end{array}\right\} \\
& Y_{2}=\left\{y \in\{0,1\}^{6} \quad \begin{array}{l}
\mid h_{2}(110011, y)+h_{1}(110011, y) \leq 2=C_{21} \\
\mid h_{2}(110011, y)+h_{3}(110011, y)=0=C_{23}
\end{array}\right\} \\
& Y_{3}=\left\{y \in\{0,1\}^{6} \begin{array}{c}
\mid h_{3}(111100, y)+h_{1}(111100, y) \leq 3=C_{31} \\
\mid h_{3}(111100, y)+h_{2}(111100, y) \leq 3=C_{32}
\end{array}\right\}
\end{aligned}
$$

Note that the worse expected payoffs in states 1 and 2 as compared to those in state 3 , make it both $C_{13}=C_{23}=0$, i.e., no Hamming distance (mistake) will be permitted between the observed $y$ and the standard prototype $\widehat{\sigma}_{1}^{S}$, if the Receiver has to assess that $y$ comes from $\widehat{\sigma}_{1}^{S}$ instead of coming from $\widehat{\sigma}_{3}^{S}$, and similarly for the standard prototype $\widehat{\sigma}_{2}^{S}$.

How to construct the pragmatic variations around the standard prototypes? Take the minimum of the $C_{l k}$ 's. In our example consider, for instance, $Y_{1}$, where this minimum is given by the Hamming distance of any output sequence $y$ to blocks 1 and 3, i.e. $h_{1}(001111, y)+$ $h_{3}(001111, y)=0$. Parameter $C_{13}=0$ implies that no error is permitted in blocks 1 and 3 together and hence these blocks in all sequences belonging to $Y_{1}$ have to be equal to the first and third blocks, respectively, of $\widehat{\sigma}_{1}^{S}=001111$, i.e. sequences of the form $\{00 * * 11\}$. Now, let us consider the elements of block 2 of $Y_{1}$, where $C_{12}=1$. This implies that at most one error is permitted in blocks 1 and 2 together, but since $C_{13}=0$, this error can only be in block 2 . Hence block 2 in all sequences in $Y_{1}$ is composed of the sequences $\{(1,1),(0,1),(1,0)\}$. Thus, block 2 permits all the variations around $\widehat{\sigma}_{1}^{S}$. The distance asymmetries among blocks reflects the expected payoffs asymmetries of $\Gamma$. Similar reasoning will give us the set of sequences in $Y=\{0,1\}^{6}$ belonging to $Y_{2}$. Finally, notice that by lemma 2 and by the integer approximation $C_{32}+C_{23}=2 m-1 \leq 2 m$ and $C_{31}+C_{13}=2 m-1 \leq 2 m$, and the sequences belonging to $Y_{3}$ can be easily characterized. 
Thus, set $Y=\{0,1\}^{6}$ is partitioned in three sets of sequences ${ }^{19} Y_{1}$, $Y_{2}$ and $Y_{3}$. where

$$
Y_{1}=\left\{\begin{array}{lll}
\{(0,0) & (1,1) & (1,1)\} \\
\{(0,0) & (0,1) & (1,1)\} \\
\{(0,0) & (1,0) & (1,1)\}
\end{array}\right\} \quad Y_{2}=\left\{\begin{array}{lll}
\{(1,1) & (0,0) & (1,1)\} \\
\{(1,0) & (0,0) & (1,1)\} \\
\{(0,1) & (0,0) & (1,1)\} \\
\{(0,0) & (0,0) & (1,1)\}
\end{array}\right\}
$$

and $Y_{3}=\{0,1\}^{6}-Y_{1}-Y_{2}$.

Then, for each $y$ the Receiver's pure equilibrium strategy is:

$$
\widehat{\sigma}_{y}^{R}=\widehat{a}_{j} \Longleftrightarrow y \in Y_{j}, j=1,2,3
$$

and equilibrium expected payoffs for $n=6$ are:

$$
\begin{aligned}
\Pi^{S}\left(\left\{\widehat{\sigma}_{j}^{S}\right\}_{j},\left\{\widehat{\sigma}_{y}^{R}\right\}_{y}\right) & =\Pi^{R}\left(\left\{\widehat{\sigma}_{j}^{S}\right\}_{j},\left\{\widehat{\sigma}_{y}^{R}\right\}_{y}\right)=\Pi_{v}=\sum_{j=1}^{3} \sum_{y \in Y^{n}} q_{j} p\left(y \mid \widehat{\sigma}_{j}^{S}\right) u\left(\widehat{\sigma}_{y}^{R}, \omega_{j}\right) \\
& =\sum_{j=1}^{3} q_{j} M_{j} \sum_{y \in Y^{n}} q_{j} p\left(y \mid \widehat{\sigma}_{j}^{S}\right)=\sum_{j=1}^{3} q_{j} p\left(Y_{j} \mid \widehat{\sigma}_{j}^{S}\right) M_{j}=10.96
\end{aligned}
$$

where $p\left(Y_{j} \mid \widehat{\sigma}_{j}^{S}\right)=\sum_{y \in Y_{j}} p\left(y \mid \widehat{\sigma}_{j}^{S}\right)$ with $p\left(Y_{1} \mid \widehat{\sigma}_{1}^{S}\right)=0.083 ; p\left(Y_{2} \mid \widehat{\sigma}_{2}^{S}\right)=$ 0.130 and $p\left(Y_{3} \mid \widehat{\sigma}_{3}^{S}\right)=0.994$. The ex-ante expected payoffs of noiseless communication are $\sum_{j=1}^{3} q_{j} M_{j}=13$ and the maximum expected payoffs of the silent game are $q_{3} M_{3}=10.75$.

Example 2. Consider the particular case $|\Omega|=2$, with $n=4$, and the alternative corpus $\widehat{\sigma}_{1}^{S}=0000$ and $\widehat{\sigma}_{2}^{S}=1111$ instead of ours (i.e, $\widehat{\sigma}_{1}^{S}=0011$ and $\left.\widehat{\sigma}_{2}^{S}=1100\right)$. Further assume that $\varepsilon_{0}=\varepsilon_{1}=\varepsilon$.

Applying our best-reply reasoning to the above corpus, the Receiver's pragmatic variations for any received sequence $y$, when $q_{1} M_{1} \neq q_{2} M_{2}$ are:

$$
\begin{aligned}
& Y_{1}=\left\{y \in\{0,1\}^{4} \quad \mid h_{1}(0000, y)+h_{2}(0000, y) \leq C_{12}\right. \\
& Y_{2}=\left\{y \in\{0,1\}^{4} \quad \mid h_{2}(1111, y)+h_{1}(1111, y) \leq C_{21}\right\}
\end{aligned}
$$

and the Receiver's pure strategy is as before: $\widehat{\sigma}_{y}^{R}=\widehat{a}_{j} \Longleftrightarrow y \in Y_{j}$, $j=1,2$.

Notice that if $\Gamma$ has symmetric expected payoffs, i.e., $q_{1} M_{1}=q_{2} M_{2}$, then $C_{12}=C_{21}=m=\frac{n}{2}$, and the Receiver's best response when she hears a $y$ will be the well-known majority rule: playing $\widehat{a}_{1}$ whenever the number of 0's is strictly greater than the number of 1's and $\widehat{a}_{2}$

\footnotetext{
${ }^{19}$ Notice that sets $Y_{1}, Y_{2}$ and $Y_{3}$ are a partition of $Y$. In order for a sequence of $Y_{3}$ to be also in $Y_{1}$, a Hamming distance of $2 m$ would be required. But the maximum distance is $2 m-1$.
} 
whenever the number of 1's is greater than or equal to the number of 0 's. Nevertheless, when $q_{1} M_{1} \neq q_{2} M_{2}$, the majority rule will not be a best-response.

\section{EfFicienCy AnAlysis}

This section analyzes the power of pragmatic Languages as coordination devices under noisy communication. This analysis entails to first assessing its performance as a meaning inference model, i.e., to bound the size of the potential wrong inferences of meaning as a function of parameters $\varepsilon$ and $n$. Then, efficiency is analyzed by comparing, for each communication length $n$, how close ex-ante expected payoffs are to those of reliable communication, thus providing, for a given expected payoff-approximation parameter, the communication threshold length.

Let $\Gamma_{v_{0}}$ be the game where the Sender communicates the realized state of nature with no mistake, i.e., $\varepsilon_{0}=\varepsilon_{1}=0$, and let $\Pi_{v_{0}}$ be the associated ex-ante Nash equilibrium expected payoffs where agents play the action pair with positive payoffs, at each state of nature, $\Pi_{v_{0}}=$ $\sum_{j=1}^{|\Omega|} q_{j} M_{j}$.

Alternatively, the common ex-ante equilibrium expected payoffs of our extended communication game $\Gamma_{v}^{n}$ were denoted by $\Pi_{v}$. To stress the dependence of such payoffs on the communication length $n$, let us denote them as $\Pi_{v}^{n}$. Also, recall that (see remark 3 before the section of examples) the vicinity bounds depend on $n$ and so do the pragmatic variations classes, denoted now as $Y_{j}^{n}, l=\{1, \ldots,|\Omega|\}$. Then,

$$
\Pi_{v}^{n}=\Pi_{v}^{n}\left(\left\{\widehat{\sigma}_{j}^{S}\right\}_{j},\left\{\widehat{\sigma}_{y}^{R}\right\}_{y}\right)=\sum_{j=1}^{|\Omega|} q_{j} M_{j} p\left(Y_{j}^{n} \mid \widehat{\sigma}_{j}^{S}\right)
$$

where $p\left(Y_{j}^{n} \mid \widehat{\sigma}_{j}^{S}\right)=\sum_{y \in Y_{j}^{n}} p\left(y \mid \widehat{\sigma}_{j}^{S}\right)$. Therefore,

$$
\Pi_{v_{0}}-\Pi_{v}^{n}=\sum_{j=1}^{|\Omega|} q_{j} M_{j}\left(1-p\left(Y_{j}^{n} \mid \widehat{\sigma}_{j}^{S}\right)\right)
$$

The difference between the above expected payoffs depends on probabilities $p\left(Y_{j}^{n} \mid \widehat{\sigma}_{j}^{S}\right)$. Each of this quantities measures the probability mass of the pragmatic variation of each standard prototype $\widehat{\sigma}_{j}^{S}$ : the Receiver's probability of playing $\widehat{a}_{j}$ when the Sender utters $\widehat{\sigma}_{j}^{S}$ in a communication episode of length $n$.

The first finding is that our pragmatic Language performs probabilistically quite well, when $n$ is not too short, as an inference meaning 
device, under noisy communication. To show this, we construct an upper bound on $1-p\left(Y_{j}^{n} \mid \widehat{\sigma}_{j}^{S}\right)=p\left(Y^{n}-Y_{j}^{n} \mid \widehat{\sigma}_{j}^{S}\right)$, i.e. the probability of not inferring the action $\widehat{a}_{j}$ by the Receiver when the Sender utters sequence $\widehat{\sigma}_{j}^{S}$. By definition of each $Y_{j}^{n}$, this wrong inference takes place whenever some vicinity bounds are not fulfilled, i.e., whenever $h_{j}\left(\widehat{\sigma}_{j}^{S}, y\right)+h_{k}\left(\widehat{\sigma}_{k}^{S}, y\right)>C_{j k}$ for $k \in K$, where $K$ is any non-empty subset in $\{1,2, \ldots, j-1, j+1, \ldots,|\Omega|\}$. Then, we partition the event $Y^{n}-Y_{j}^{n}$ into a series of disjoint events $E_{K}$, where each of them is formed by the output sequences not satisfying the corresponding vicinity bounds $C_{j k}$ for $k \in K$. In the Appendix it is proven ${ }^{20}$ that the probability of such events, $p\left(E_{K} \mid \widehat{\sigma}_{j}^{S}\right)$, and then $p\left(Y^{n}-Y_{j}^{n} \mid \widehat{\sigma}_{j}^{S}\right)$, can be written as a polynomial of the bigger noise parameter, say $\varepsilon_{1}$, and the smallest vicinity bound (which depends on $n$ ). The next Proposition states this result:

Proposition 2. Given a noisy communication channel $v\left(\varepsilon_{0}, \varepsilon_{1}\right)$ with $\varepsilon_{0}<\varepsilon_{1}$ and game $\Gamma_{v}^{n}$, for any $n \geq|\Omega|$, then

$$
p\left(Y^{n}-Y_{j}^{n} \mid \widehat{\sigma}_{j}^{S}\right)=1-p\left(Y_{j}^{n} \mid \widehat{\sigma}_{j}^{S}\right) \leq \varepsilon_{1}^{c_{j}+1} \phi_{j}\left(\varepsilon_{0}, \varepsilon_{1}\right)
$$

where $c_{j}=\min \left\{C_{j l}|l=1, \ldots,| \Omega \mid ; j \neq l\right\}$ and $\phi\left(\varepsilon_{0}, \varepsilon_{1}\right)$ is a function on $\varepsilon_{0}$ and $\varepsilon_{1}$ such that $0 \leq \phi_{j}\left(\varepsilon_{0}, \varepsilon_{1}\right) \leq 1$, for each $j$.

The above Proposition says that there is an upper bound on the probability of wrong inferences. The formula precisely measures such a bound $^{21}$. Notice that this bound is small whenever $c_{j} \geq 0$. A sufficient condition for $c_{j} \geq 0$ is that all vicinity bounds $C_{l k}$ are non-negative,

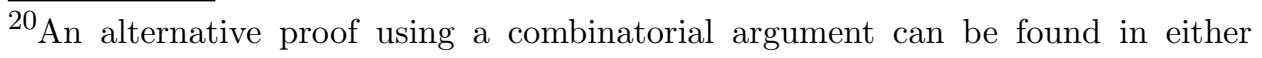
http://www.uv.es/penelope/ or http://www.uv.es/urbano/ .

${ }^{21}$ This bound allows us to obtain asymptotic properties of the proposed equilibrium strategies. By the above proposition, $1 \geq p\left(Y_{j}^{n} \mid \widehat{\sigma}_{j}^{S}\right) \geq 1-\varepsilon_{1}^{c_{j}^{m}+1} \phi_{n}\left(\varepsilon_{0}, \varepsilon_{1}\right)$, where $c_{j}^{m}$ is a function of $n$, since $m=\frac{n}{|\Omega|}$ and polynomials $\phi_{n}\left(\varepsilon_{0}, \varepsilon_{1}\right)$ also depend on $n$. By remark $3, c_{j}^{m}$ grows by a unit whenever the block length $m=\frac{n}{|\Omega|}$ increases by one. Then, since $\varepsilon_{0}, \varepsilon_{1}<1$, then $\lim _{n \rightarrow \infty} \varepsilon_{1}^{c_{j}+1}=0$ and $\lim _{n \rightarrow \infty} \phi_{n}\left(\varepsilon_{0}, \varepsilon_{1}\right)$ is a constant:

$$
\lim _{n \rightarrow \infty} p\left(Y_{j}^{n} \mid \widehat{\sigma}_{j}^{S}\right) \geq 1-\lim _{n \rightarrow \infty} \varepsilon_{1}^{c_{j}^{m}+1} \phi_{n}\left(\varepsilon_{0}, \varepsilon_{1}\right)=1
$$

Therefore, the limit of $\left(\Pi_{v_{0}}-\left\{\Pi_{v}^{n}\right\}_{n}\right)$ is 0 when $n$ goes to infinity, since this limit is $q_{j} M_{j}-\lim _{n \rightarrow \infty} q_{j} M_{j}\left\{p\left(Y_{j}^{n} \mid \widehat{\sigma}_{j}^{S}\right)\right\}_{n}$ and it is zero, for all $j=1, \ldots,|\Omega|$, whenever $\lim _{n \rightarrow \infty}\left\{p\left(Y_{j}^{n} \mid \widehat{\sigma}_{j}^{S}\right)\right\}_{n}=1$.
} 
i.e., whenever,

$$
n \geq-|\Omega| \frac{\ln \frac{q_{l} M_{l \mid}}{q_{j} M_{j}}}{\ln \frac{\varepsilon_{0} \varepsilon_{1}}{\left(1-\varepsilon_{0}\right)\left(1-\varepsilon_{1}\right)}}, \text { for all } l=1, \ldots,|\Omega| ; j \neq l .
$$

We turn next to the efficiency issue. For a fixed communication length $n$, a pair of equilibrium strategies $\left(\left\{\widehat{\sigma}_{j}^{S}\right\}_{j},\left\{\widehat{\sigma}_{y}^{R}\right\}_{y}\right)$ is $\eta$-efficient if: $\Pi_{v_{0}}-\Pi_{v}^{n} \leq \eta$.

For any $1>\eta>0$, we offer a threshold length $\widehat{n}$ such that both the associated corpus and set of pragmatic variations support $\eta$-efficient equilibrium strategies.

By Proposition 2 and since $0 \leq \phi_{j}\left(\varepsilon_{0}, \varepsilon_{1}\right) \leq 1$, the difference between reliable and noisy communication expected payoffs is given by

$\Pi_{v_{0}}-\Pi_{v}^{n}=\sum_{j=1}^{|\Omega|} q_{j} M_{j}\left(1-p\left(Y_{j}^{n} \mid \widehat{\sigma}_{j}^{S}\right)\right)=\sum_{j=1}^{|\Omega|} q_{j} M_{j} \varepsilon_{1}^{c_{j}+1} \phi_{j}\left(\varepsilon_{0}, \varepsilon_{1}\right) \leq \sum_{j=1}^{|\Omega|} q_{j} M_{j} \varepsilon_{1}^{c_{j}+1}$ where $c_{j}=\min \left\{C_{j l}|l=1, \ldots,| \Omega \mid ; j \neq l\right\}$. Denote $\widetilde{c}=\min \left\{c_{j} \mid j=\right.$ $1, \ldots,|\Omega|\}$, and assume, without loss of generality, that $q_{1} M_{1} \leq \ldots \leq$ $q_{|\Omega|} M_{|\Omega|}$, then the smallest vicinity bound $\widetilde{c}$ is

$$
\widetilde{c}=\frac{\ln \frac{q_{|\Omega|} M_{|\Omega|}}{q_{1} M_{1}}}{\ln \frac{\varepsilon_{0} \varepsilon_{1}}{\left(1-\varepsilon_{0}\right)\left(1-\varepsilon_{1}\right)}}+\frac{n}{|\Omega|}
$$

Therefore, to get an $\eta$-approximation, it suffices that

$$
\Pi_{v_{0}}-\Pi_{v}^{n} \leq \varepsilon_{1}^{\widetilde{c}+1} \sum_{j=1}^{|\Omega|} q_{j} M_{j} \leq \eta
$$

Or, in other words,

$$
\varepsilon_{1}^{\widetilde{c}+1} \leq \frac{\eta}{\sum_{j=1}^{|\Omega|} q_{j} M_{j}}
$$

Since $\ln \varepsilon_{1}<0$, a sufficient condition is that

$$
\widetilde{c} \geq \frac{1}{\ln \varepsilon_{1}} \ln \frac{\eta}{\sum_{j=1}^{|\Omega|} q_{j} M_{j}}>\frac{1}{\ln \varepsilon_{1}} \ln \frac{\eta}{\sum_{j=1}^{|\Omega|} q_{j} M_{j}}-1 .
$$

Inserting (5) in (6) and rearranging we find that $\widehat{n}$ is bounded by the expression,

$$
\widehat{n} \geq|\Omega|\left(\frac{1}{\ln \varepsilon_{1}} \ln \frac{\eta}{\sum_{j=1}^{|\Omega|} q_{j} M_{j}}-\frac{\ln \frac{q_{|\Omega|} M_{|\Omega|}}{q_{1} M_{1}}}{\ln \frac{\varepsilon_{0} \varepsilon_{1}}{\left(1-\varepsilon_{0}\right)\left(1-\varepsilon_{1}\right)}}\right) .
$$


The minimum length of the communication episode that allows $\eta$ efficiency depends on the relative $\eta$-approximation, the biggest amount of noise and the maximum relative expected payoffs loss. Notice that by (4) if the first term of the right hand side of (7) were negligible, then the length $\widehat{n}$ would coincide with the minimal length guaranteeing a positive matrix of the $C_{l k}$ 's. Therefore, the second term of the right hand side is the communication length to generate non-empty pragmatic variations for all the prototypes. The first term adds then the time needed to span such pragmatic variations in order to reduce the chances of misunderstandings and increase expected payoffs according to the $\eta$-efficiency.

The intuition is clear, as $n$ increases the vicinity bounds increase as well, spanning the pragmatic variation classes $Y_{l}, l=\{1, \ldots,|\Omega|\}$. Therefore, the communication length $n$ has to be long enough to guarantee that, on one hand, there are not efficiency losses due to empty pragmatic variation classes, and on the other, the probability masses of the pragmatic variation classes are sufficiently high to achieve exante expected payoffs $\eta$-close to those of reliable communication. This result is stated below.

Theorem 2. Let $\eta>0$, for any communication length $n \in[\widehat{n}, \infty)$,

$$
\Pi_{v_{0}}-\Pi_{v}^{n} \leq \eta
$$

We would like to remark that although the corpus works quite efficiently in most of the cases, there may exist situations where communication is so short that some prototypes may be unable to generate a meaning. This creates inefficiencies that could be easily avoided by a reassignment of the signals. More precisely, in the corpus construction, $\frac{n}{|\Omega|}$ out of the available $n$ signals, those equal to 0 , are used to distinguish each standard prototype from any other else. If there exists a state $\omega_{j}$ such that $C_{j k}<0$ for any $k=1, \ldots,|\Omega|, k \neq j$, then $Y_{j}=\varnothing$ and $p\left(Y_{j} \mid \widehat{\sigma}_{j}^{S}\right)=0$. In this case, the Receiver's action $\widehat{a}_{j}$ will never be chosen and the $\frac{n}{|\Omega|}$ signals devoted to distinguish $\widehat{\sigma}_{j}^{S}$ from the other prototypes will be wasted. To avoid this inefficiency, the corpus is modified such that the 0's signals used in those prototypes sequences such that $p\left(Y_{j} \mid \widehat{\sigma}_{j}^{S}\right)=0$ are reassigned to the other prototypes. 
Example 4. Consider the incomplete information Sender-Receiver game with $\Omega=\left\{\omega_{1}, \omega_{2}, \omega_{3}\right\}$, where nature chooses $\omega_{j}$ according to law $q=\left(q_{1}, q_{2}, q_{3}\right)=(0.1,0.3,0.6)$. Payoffs for the three states of nature are $M_{1}=3, M_{2}=20$ and $M_{3}=100$.

Consider the matrix of parameters $C_{l k}-m$,

$$
\left(\begin{array}{ccc}
* & 1 & 2 \\
-2 & * & 1 \\
-3 & -2 & *
\end{array}\right)
$$

For $n=3$, then $m=\frac{n}{|\Omega|}=1$, and the sets of pragmatic variations $Y_{1}$ and $Y_{2}$ are empty, the corresponding actions $\widehat{a}_{1}, \widehat{a}_{2}$ will never be chosen and the communication game will be equivalent to the silent game, where no-communication takes place. On the other hand, for $n \geq 9$, and then $m \geq 3$, all the pragmatic variations are non-empty and the Receiver's three actions will be played with ex-ante positive probability.

For the intermediate value of $n=6(m=2)$, the matrix of vicinity bounds, $C_{l k}$, after integer approximations, is:

$$
\left.\begin{array}{r}
k=1 \\
k=2 \\
k=3
\end{array} \quad \begin{array}{rrr}
C_{1 k} & C_{2 k} & C_{3 k} \\
* & 3 & 4 \\
0 & * & 3 \\
-1 & 0 & *
\end{array}\right)
$$

In this case the prototype $\widehat{\sigma}_{1}^{S}=001111$ is unable to generate a meaning (the corresponding Receiver's action $\widehat{a}_{1}$ ) and the two 0-signals of the sequence devoted to distinguish it from the others are wasted. To avoid this inefficiency, players could act as if they were playing another (truncated) game, with only two states of nature $\omega_{2}$ and $\omega_{3}$, each of them taking place with probabilities $\widetilde{q}_{2}=q_{2}+q_{1} \frac{q_{2}}{q_{2}+q_{3}}$ and $\widetilde{q}_{3}=q_{3}+$ $q_{1} \frac{q_{3}}{q_{2}+q_{3}}$, respectively. In this case the new corpus consists of the two standard prototypes $\widetilde{\sigma}_{2}^{S}=000111, \widetilde{\sigma}_{3}^{S}=111000$ and no signal is wasted now.

To formalize this idea, consider game $\Gamma$, with $\Omega=\left\{\omega_{1}, \ldots, \omega_{|\Omega|}\right\}$, where $\omega_{j}$ is chosen by nature with probability $q_{j}$, and its communication extension by adding $n$ uses of the noisy channel $v$, denoted by $\Gamma_{v}^{n}$. Let 
as assume, without loss of generality, that $p\left(Y_{1} \mid \widehat{\sigma}_{1}^{S}\right) \geq p\left(Y_{2} \mid \widehat{\sigma}_{2}^{S}\right) \geq \ldots \geq$ $p\left(Y_{|\Omega|} \mid \widehat{\sigma}_{|\Omega|}^{S}\right)$ and let $j_{0}=\min \left\{j=1, \ldots,|\Omega| \mid p\left(Y_{j} \mid \widehat{\sigma}_{j}^{S}\right)>0\right\}$.

Given $\Gamma$ and $\Gamma_{v}^{n}$, define the auxiliary truncated game $\Gamma=\left\{\Gamma_{1}, \ldots, \Gamma_{j_{0}}\right\}$ where nature chooses state $\omega_{j}$ (and the the game $\Gamma_{j}$ ) with probability

$$
\widetilde{q}_{j}=q_{j}+\frac{q_{j}}{\sum_{l=0}^{j_{0}} q_{l}} \sum_{k=j_{0}+1}^{|\Omega|} q_{k} \geq q_{j}
$$

for $j=1, \ldots, j_{0}$.

Let $\widetilde{\Gamma}_{v}^{n}$ the corresponding extended game and $\widetilde{\sigma}_{j}^{S}, j=1, \ldots, j_{0}$, the standard prototypes of the new corpus. Since $p\left(Y_{j} \mid \widetilde{\sigma}_{j}^{S}\right) \geq p\left(Y_{j} \mid \widehat{\sigma}_{j}^{S}\right)$ for $j=1, \ldots, j_{0}$ and $p\left(Y_{j} \mid \widehat{\sigma}_{j}^{S}\right)=0$ for $j=j_{0}+1, \ldots,|\Omega|$, we have that $\widetilde{\Pi}_{v}^{n}=\sum_{j=1}^{j_{0}} \widetilde{q}_{j} M_{j} p\left(Y_{j}^{n} \mid \widetilde{\sigma}_{j}^{S}\right) \geq \sum_{j=1}^{j_{0}} q_{j} M_{j} p\left(Y_{j}^{n} \mid \widehat{\sigma}_{j}^{S}\right)=\sum_{j=1}^{|\Omega|} q_{j} M_{j} p\left(Y_{j}^{n} \mid \widehat{\sigma}_{j}^{S}\right)=\Pi_{v}^{n}$ and no message is wasted trying to distinguish among actions that will never be chosen.

\section{Concluding Remarks}

We have shown that pragmatic Languages with a universal grammar are a powerful coordination device when there may exist communication misunderstandings. Reduced dictionaries ${ }^{22}$, simple grammars and standard prototypes help individuals to coordinate in spite of initial misunderstandings. This is accomplished by facilitating the inference of meaning and thus generating the pragmatic variations around each standard prototype. Our approach sheds light to the formation not only of target-oriented languages, but also to specific "organization" languages, professional languages, etc.

When considering real life time-constraints, a language with structure based on different orderings of the enumerations turns out to be more useful for learning purposes rather than for meaning inference. Nevertheless, languages with universal grammars appear to have emerged because they ensure the successful transmission of languages

\footnotetext{
${ }^{22}$ Nowak, Krakauer and Dress (1999) argue that, because of the noise, the fitness of a language cannot be increased arbitrarily by just adding more signals. On the contrary, the fitness can be increased by combining a small number of signal into words. This is called "phonemes" by linguists. Modern human languages have a limited number of phonemes: as reported by Nowak, Krakauer and Dress, all of 317 languages in the University of California Los Angeles Segment Inventory Database (UPSID) have between 11 and 141 phonemes, but $70 \%$ of these languages have between 20 and 37 phonemes.
} 
themselves. The Chinese Language is an example of how the successful transmission of information shapes some language characteristics. Spoken Chinese is distinguished by its high level of internal diversity (it is pragmatic and very local) though all spoken varieties of Chinese ${ }^{23}$ are tonal and analytic; dictionaries are small with 6 vowels and 15 consonants and the grammar is compositional. On the contrary, written Chinese is highly complex: it comprises the written symbols used to represent spoken Chinese. Chinese characters do not constitute an alphabet or a compact syllabary; they are instead built up from simple parts representing objects or abstracts notions. There are around 47.035 ideograms or hanzy, but Chinese people do not manage more than 8000 of them.

One of the frequently asked questions in studies on language origins and evolution is how universal grammar structures in human languages could have emerged. One line of research assumes that such structures emerged from exploiting regularities found in protolanguages. Universal structures in language could have emerged when the learning examples do not cover the entire language (i.e., there was a bottleneck on the transmission of language). Other researchers have assumed that the ability to use syntax has evolved as a biological adaptation. In their seminal article which re-ignited much of recent burgeoning interest in language evolution, Pinker and Bloom (1990) argue persuasively that "a specialization for grammar evolved by a conventional neo-Darwinian process" (page 707), suggesting that humans have evolved an innate, genetically specified module in the brain, which specifies a formal coding of the principles of Universal Grammar. These authors are firmly of the opinion that the selective advantage of the communicative function of language can explain the evolution of the language faculty itself. But, Chomsky (1988), perhaps somewhat surprisingly, given his introduction of the very idea of Universal Grammar, argues that the role of natural selection in language evolution is very limited. Much more effort in computer simulations of language evolution has to be done to give more precise answers.

To conclude, we would like to call the attention about the way of precisely defining the notions of a language and a "common language" from

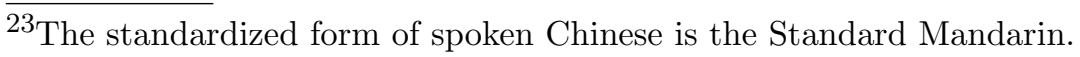


an economic viewpoint. As it is obvious, different models may need different notions of both languages and common languages. Therefore, much work is needed to define some unifying rules which are still lacking.

\section{REFERENCES}

Azrieli, Y. and E. Lehrer (2007): "Categorization generated by extended prototypes: An axiomatic approach", Journal of Mathematical Psychology 51, 14-28

Balinski, M. and R. Laraki (2007,a): "Election by Majority Judgement: Experimental Evidence", Cahier du Laboratoire d' Econometrie, École Polythecnique, no 2007-28.

Balinski, M. and R. Laraki (2007,b): "A theory of measuring, electing and ranking", Proceedings of the National Academy of Sciences, U.S.A., vol. 104(2007), 8720-8725.

Batali, J. (1998): "Computational simulations of the emergence of grammar". In J.R. Hurford, M. Studdert-Kennedy, and C. Knight (editors), Approaches to the evolution of language: Social and cognitive bases, 405-426. Cambridge, Cambridge University Press.

Blume, A. (2005): "A Learning-Efficiency Explanation of Structure in Language", Theory and Decision, 57, 265-285.

Blume, A. and O. J. Board (2008): "Intentional Vagueness", mimeo.

Blume, A., O. J. Board and K. Kawamura (2007): "Noisy Talk", Theoretical Economics, Vol. 2, 395-440.

Chomsky, N. (1988): Language and problems of knowledge, Cambridge, MA. The MIT Press.

Crawford, V. and J. Sobel (1982): "Strategic Information Transmission", Econometrica, Vol. 50, 1431-1451.

Crémer, J., L. Garicano and A. Prat (2007): "Language and the Theory of the Firm", Quarterly Journal of Economics, 122(1), 373408.

Demichelis, S and J. Weibull (2008): "Language, meaning and games: A model of communication, coordination and evolution", American Economic Review, forthcoming.

Gärdenfors, P. (2000): "Conceptual Spaces". Cambridge, MA: The MIT Press. 
Grice, H.P. (1975): "Logic and Conversation". In P. Cole and J.L. Morgan (editors), Syntax and semantics, vol. 3, 41-58. New York. Academic Press.

Jäger, G. (2007): "The evolution of convex categories", Linguist and Philos, 30, 551-564. Published online: 26 March 2008.

Hernández, P., Urbano, A. and J. Vila (2009): "Nash Equilibrium and information transmission coding and decoding rules", mimeo, University of Valencia.

Kirby, S. (2002): "Learning, bottlenecks and the evolution of recursive syntax". In E. Bricoe, editor, Linguistics evolution through language acquisition: Formal and Computational models, 173-203, Cambridge, Cambridge University Press.

Kirby, S. (2007): "The evolution of the meaning-espace structure through iterated learning", In Lyon, C., Nehaniv, C., and Cangelosi, A., editors, Emergence of Communication and Language, 253-268, SpringVerlang.

Koessler, F. (2001): "Common Knowledge and Consensus with Nosy Communication", Mathematical Social Sciences, 42(2), 139-159.

Koessler, F. (2004): "Strategic Knowledge Sharing in Bayesian Games", Games and Economic Behavior, 48(2), 292-320.

Lipman, B., and D. Seppi (1995): "Robust Inference in Communication Games with Partial Peovability", Journal of Economic Theory 66, 370-405.

Nowak, M. A. and D. C. Krakauer (1999): "The evolution of language", Proc. Natl. Acad. Sci. USA, vol. 96, 8028-8033.

Nowak, M. A., D.C. Krakauer and A. Dress (1999): "An error limit for the evolution of language", Proceedings of the Royal Society B: Biological Sciences, 266, 2131-2136.

Pinker, S and P. Bloom (1990): "Natural language and natural selection", Behavioral and Brain Sciences, 13, 707-784.

Reiter, E. and S. Sripada (2002): "Human Variation and Lexical Choice", Association for Computational Linguistics, 28, 545-553.

Rubinstein, A. (1996): "Why are certain properties of binary relations relatively more common in natural language, Econometrica, Vol. 64, 343-355. 
Rubinstein, A. (2000): Economics and Language, Cambridge University Press, Cambridge.

Selten, R.and M. Warglien (2007): "The emergence of simple languages in an experimental coordination game", PNAS, vol. 104 (18), 7361-7366.

Shannon, C.E. (1948): "A Mathematical Theory of Communication," Bell System Technical Journal, 27, 379-423; 623-656.

Smith, A.D.M. (2003): Evolving Communication through the inference of Meaning, $\mathrm{PhD}$ thesis, University of Edinburg.

Vogt, P. (2005): "The emergence of compositional structures in perceptually grounded language games", Artificial Intelligence 167(1-2): 206-242.

\section{ApPENDix}

Proof of Lemma 1: If $\varepsilon_{0}+\varepsilon_{1}<1$, then $p(1 \mid 0)=\varepsilon_{0}<1-\varepsilon_{1}=$ $p(1 \mid 1)$ and $p(0 \mid 1)=\varepsilon_{1}<1-\varepsilon_{0}=p(0 \mid 0)$. Clearly, the conditional probability of receiving a 0 , when a 0 was sent is higher than the one of receiving a 0 when a 1 was sent, therefore, $\frac{p(0 \mid 0)}{p(0 \mid 1)} \neq 1$. And similarly for the conditional probability of receiving a 1 , i.e., $\frac{p(1 \mid 1)}{p(1 \mid 0)} \neq 1$. Thus, information transmission is informative since $p(r \mid s) \neq p(r \mid \widehat{s})$ for any $r \in\{0,1\}$ and $s, \widehat{s} \in\{0,1\}$ and thus,

$$
\frac{p(s \mid r)}{p(\widehat{s} \mid r)}=\frac{p(r \mid s) p(s)}{p(r \mid \widehat{s}) p(\widehat{s})} \neq \frac{p(s)}{p(\widehat{s})}
$$

Now, let $r=0$ and $\widehat{r}=1, s=0$ and $\widehat{s}=1$, then $p(r \mid s)=p(0 \mid 0)=1-$ $\varepsilon_{0}, p(\widehat{r} \mid \widehat{s})=p(1 \mid 1)=1-\varepsilon_{1}, p(r \mid \widehat{s})=p(0 \mid 1)=\varepsilon_{1}$ and $p(\widehat{r} \mid s)=p(1 \mid 0)=$ $\varepsilon_{0}$. Let us check that output signal 0 is more favorable than output signal 1 , for input signal 0 :

$$
\frac{p(r \mid s)}{p(r \mid \widehat{s})}=\frac{p(0 \mid 0)}{p(0 \mid 1)}=\frac{\left(1-\varepsilon_{0}\right)}{\varepsilon_{1}}>\frac{\varepsilon_{0}}{\left(1-\varepsilon_{1}\right)}=\frac{p(1 \mid 0)}{p(1 \mid 1)}=\frac{p(\widehat{r} \mid s)}{p(\widehat{r} \mid \widehat{s})}
$$

Similarly, letting now $r=1$ and $\widehat{r}=0, s=1$ and $\widehat{s}=0$, then $p(r \mid s)=p(1 \mid 1)=1-\varepsilon_{1}, p(\widehat{r} \mid \widehat{s})=p(0 \mid 0)=1-\varepsilon_{0}, p(r \mid \widehat{s})=p(1 \mid 0)=\varepsilon_{0}$ and $p(\widehat{r} \mid s)=p(0 \mid 1)=\varepsilon_{1}$. As above, since $\left(1-\varepsilon_{0}\right)\left(1-\varepsilon_{1}\right)>\varepsilon_{1} \varepsilon_{0}$, then $p(r \mid s) p(\widehat{r} \mid \widehat{s})>p(r \mid \widehat{s}) p(\widehat{r} \mid s)$ and output signal 1 is more favorable than output signal 0 , for input signal 1.

If $\varepsilon_{0}+\varepsilon_{1}>1$, then $p(1 \mid 0)=\varepsilon_{0}>1-\varepsilon_{1}=p(1 \mid 1)$ and $p(0 \mid 1)=\varepsilon_{1}>$ $1-\varepsilon_{0}=p(0 \mid 0)$. In words, the conditional probability of receiving a 0 , 
when a 0 was sent is lower than the one of receiving a 0 when a 1 was sent. As above, it can be easily proven again that information transmission is informative: i.e., $\frac{p(s \mid r)}{p(\widehat{s} \mid r)}=\frac{p(r \mid s) p(s)}{p(r \mid \widehat{s}) p(\widehat{s})} \neq \frac{p(s)}{p(\widehat{s})}$, and that output signal 0 is now more favorable than output signal 1 , for input signal 1.

Finally notice that when $\varepsilon_{0}+\varepsilon_{1}=1$, then $p(1 \mid 0)=\varepsilon_{0}=1-\varepsilon_{1}=$ $p(1 \mid 1)$ and $p(0 \mid 1)=\varepsilon_{1}=1-\varepsilon_{0}=p(0 \mid 0)$. Now input signals are not informative at all, since the conditional probability of receiving a 0 , when a 0 was sent is equal to the one of receiving a 0 when a 1 was sent.

Proof of Lemma 2: 1) Recall that $\widehat{\sigma}_{k}^{S}=\left\{x_{k}^{j}\right\}_{j \in\{1, \ldots, n\}}$ and $\widehat{\sigma}_{k^{\prime}}^{S}=$ $\left\{x_{k^{\prime}}^{j}\right\}_{j \in\{1, \ldots, n\}}$ where $x_{k}^{j}=x_{k^{\prime}}^{j}=1$ but the blocks $k$ and $k^{\prime}$ (i.e. $j$ such that $(i-1) m-1 \leq j \leq i m$ for $\left.i \in\left\{k, k^{\prime}\right\}\right)$. Therefore, the Hamming distance in the block $l$ is:

$$
\begin{aligned}
h_{l}\left(\widehat{\sigma}_{k}^{S}, y\right) & =\sum_{j=1}^{m} I_{y_{(l-1) m+j} \neq\left(\widehat{\sigma}_{k}^{S}\right)_{(l-1) m+j}}=\sum_{j=1}^{m} I_{y_{(l-1) m+j} \neq 1} \\
& =\sum_{j=1}^{m} I_{y_{(l-1) m+j} \neq\left(\widehat{\sigma}_{k^{\prime}}^{S}\right)_{(l-1) m+j}}=h_{l}\left(\widehat{\sigma}_{k^{\prime}}^{S}, y\right)
\end{aligned}
$$

2) Let us compute $h_{k}\left(\widehat{\sigma}_{k}^{S}, y\right)+h_{k}\left(\widehat{\sigma}_{k^{\prime}}^{S}, y\right)$ if $k \neq k^{\prime}$. Notice that:

$$
\begin{aligned}
\left\{x_{k}^{j}\right\}_{j \in\{(k-1) m+1, \ldots, k m\}}= & 0 \text { and }\left\{x_{k^{\prime}}^{j}\right\}_{j \in\{(k-1) m+1, \ldots, k m\}}=1, \text { then } \\
h_{k}\left(\widehat{\sigma}_{k}^{S}, y\right)+h_{k}\left(\widehat{\sigma}_{k^{\prime}}^{S}, y\right) & =h\left((0, \ldots, 0),\left(y^{(k-1) m+1}, \ldots, y^{k m}\right)\right) \\
& +h\left((1, \ldots, 1),\left(y^{(k-1) m+1}, \ldots, y^{k m}\right)\right) \\
& =h\left((0, \ldots, 0),\left(y^{(k-1) m+1}, \ldots, y^{k m}\right)\right) \\
& +m-h\left((0, \ldots, 0),\left(y^{(k-1) m+1}, \ldots, y^{k m}\right)\right) \\
& =m
\end{aligned}
$$

Proof that sets $Y_{l}, l=\{1, \ldots,|\Omega|\}$, are a true partition of $Y^{n}$ : Given the description of $Y_{l}$ in the main text, without loss of generality, suppose on the contrary that $Y_{1} \cap Y_{2} \neq \emptyset$.

a. Let $1 \in \tilde{\Omega}_{2}$ and $y \in Y_{1} \cap Y_{2}$. Then,

$$
h_{1}\left(\widehat{\sigma}_{1}^{S}, y\right)+h_{2}\left(\widehat{\sigma}_{1}^{S}, y\right) \leq C_{12} \text {, and } h_{2}\left(\widehat{\sigma}_{2}^{S}, y\right)+h_{1}\left(\widehat{\sigma}_{2}^{S}, y\right) \leq C_{21}
$$

and adding $h_{1}\left(\widehat{\sigma}_{1}^{S}, y\right)+h_{1}\left(\widehat{\sigma}_{2}^{S}, y\right)+h_{2}\left(\widehat{\sigma}_{1}^{S}, y\right)+h_{2}\left(\widehat{\sigma}_{2}^{S}, y\right) \leq C_{12}+C_{21}$, that by Lemma $1(2)$ is $m+m \leq 2 m-1$, a contradiction.

b. Let $1 \notin \tilde{\Omega}_{2}$ and $y \in Y_{1} \cap Y_{2}$.

$$
h_{1}\left(\widehat{\sigma}_{1}^{S}, y\right)+h_{2}\left(\widehat{\sigma}_{1}^{S}, y\right) \leq C_{12} \text {, and } h_{2}\left(\widehat{\sigma}_{2}^{S}, y\right)+h_{1}\left(\widehat{\sigma}_{2}^{S}, y\right)<C_{21}
$$


and adding $h_{1}\left(\widehat{\sigma}_{1}^{S}, y\right)+h_{1}\left(\widehat{\sigma}_{2}^{S}, y\right)+h_{2}\left(\widehat{\sigma}_{1}^{S}, y\right)+h_{2}\left(\widehat{\sigma}_{2}^{S}, y\right)<C_{12}+C_{21}$, that by Lemma $1(2)$ is $2 m<2 m$, a contradiction again.

Proof of Proposition 1: By Bayes's Theorem, $\frac{p\left(\widehat{\sigma}_{l}^{S} \mid y\right)}{p\left(\widehat{\sigma}_{k}^{S} \mid y\right)}=\frac{\frac{p\left(y||_{l}^{S}\right) c\left(\widehat{\sigma}_{l}^{S}\right)}{p(y)}}{\frac{p\left(y||_{k}^{S}\right) p\left(\widehat{\sigma}_{k}^{S}\right)}{p(y)}}=$ $\frac{q_{l}}{q_{k}} \frac{p\left(y \mid \widehat{\sigma}_{l}^{S}\right)}{p\left(y \mid \widehat{\sigma}_{k}^{S}\right)}$.

The conditional probability of the channel to generate output $y$ if message $\widehat{\sigma}_{l}^{S}$ is sent can be written as:

$$
\begin{aligned}
p\left(y \mid \widehat{\sigma}_{l}^{S}\right) & =\prod_{t=1}^{n} p\left(y_{t} \mid\left(\widehat{\sigma}_{l}^{S}\right)_{t}\right)=\prod_{t=1}^{l m} p\left(y_{t} \mid 1\right) \prod_{t=l m+1}^{(l+1) m} p\left(y_{t} \mid 0\right) \prod_{t=(l+1) m+1}^{n} p\left(y_{t} \mid 1\right) \\
& =\varepsilon_{0}^{h_{l}\left(\widehat{\sigma}_{l}^{S}, y\right)}\left(1-\varepsilon_{0}\right)^{\left.m-h_{l}\left(\widehat{\sigma}_{l}^{S}, y\right)\right)} \prod_{\substack{\alpha=1 \\
\alpha \neq l}}^{|\Omega|}\left[\varepsilon_{1}^{h_{\alpha}\left(\widehat{\sigma}_{l}^{S}, y\right)}\left(1-\varepsilon_{1}\right)^{m-h_{\alpha}\left(\widehat{\sigma}_{l}^{S}, y\right)}\right]
\end{aligned}
$$

and the conditional probability to generate the same $y$ if message $\widehat{\sigma}_{k}^{S}$ is sent instead is:

$$
p\left(y \mid \widehat{\sigma}_{k}^{S}\right)=\varepsilon_{0}^{h_{k}\left(\widehat{\sigma}_{k}^{S}, y\right)}\left(1-\varepsilon_{0}\right)^{m-h_{k}\left(\widehat{\sigma}_{k}^{S}, y\right)} \prod_{\substack{\alpha \neq k \\ \alpha=1}}^{|\Omega|}\left[\varepsilon_{1}^{h_{\alpha}\left(\widehat{\sigma}_{k}^{S}, y\right)}\left(1-\varepsilon_{1}\right)^{m-h_{\alpha}\left(\widehat{\sigma}_{k}^{S}, y\right)}\right]
$$

Consider the likelihood ratio of messages $\widehat{\sigma}_{l}^{S}$ and $\widehat{\sigma}_{k}^{S}$, when $y$ is realized, $\frac{p\left(\widehat{\sigma}_{l}^{S} \mid y\right)}{p\left(\widehat{\sigma}_{k}^{S} \mid y\right)}$. It is not difficult to show by some cumbersome algebra that this ratio can be splitted in three separated terms.

$$
\frac{p\left(\widehat{\sigma}_{l}^{S} \mid y\right)}{p\left(\widehat{\sigma}_{k}^{S} \mid y\right)}=\frac{q_{l}}{q_{k}} \times \text { Ratio }_{1} \times \text { Ratio }_{2} \times \text { Ratio }_{3}, \text { where }
$$

Ratio $_{1}$ : Measures the probability of transformation of the 0's bits in block $l$ with respect to the corresponding probability in block $k$. By lemma 2 part 2 ,

$$
\begin{aligned}
\text { Ratio }_{1} & =\frac{\varepsilon_{0}^{h_{l}\left(\widehat{\sigma}_{l}^{S}, y\right)}\left(1-\varepsilon_{0}\right)^{m-h_{l}\left(\widehat{\sigma}_{l}^{S}, y\right)}}{\varepsilon_{0}^{h_{k}\left(\widehat{\sigma}_{k}^{S}, y\right)}\left(1-\varepsilon_{0}\right)^{m-h_{k}\left(\widehat{\sigma}_{k}^{S}, y\right)}}=\frac{\varepsilon_{0}^{h_{l}\left(\widehat{\sigma}_{l}^{S}, y\right)}\left(1-\varepsilon_{0}\right)^{m-h_{l}\left(\widehat{\sigma}_{l}^{S}, y\right)}}{\varepsilon_{0}^{m-h_{k}\left(\widehat{\sigma}_{l}^{S}, y\right)}\left(1-\varepsilon_{0}\right)^{h_{k}\left(\widehat{\sigma}_{l}^{S}, y\right)}} \\
& =\left(\frac{\varepsilon_{0}}{1-\varepsilon_{0}}\right)^{h_{l}\left(\widehat{\sigma}_{l}^{S}, y\right)+h_{k}\left(\widehat{\sigma}_{l}^{S}, y\right)-m}
\end{aligned}
$$

Ratio $_{2}$ : Refers to the probability of transformation of the 1's bits in block $k$ with respect to the corresponding probability in block $l$. Then, by lemma lemma2 part 2 :

$$
\begin{aligned}
\text { Ratio }_{2} & =\frac{\varepsilon_{1}^{h_{k}\left(\widehat{\sigma}_{l}^{S}, y\right)}\left(1-\varepsilon_{1}\right)^{m-h_{k}\left(\widehat{\sigma}_{l}^{S}, y\right)}}{\varepsilon_{1}^{h_{l}\left(\widehat{\sigma}_{k}^{S}, y\right)}\left(1-\varepsilon_{1}\right)^{m-h_{l}\left(\widehat{\sigma}_{k}^{S}, y\right)}}=\frac{\varepsilon_{1}^{m-h_{l}\left(\widehat{\sigma}_{l}^{S}, y\right)}\left(1-\varepsilon_{1}\right)^{h_{k}\left(\widehat{\sigma}_{l}^{S}, y\right)}}{\varepsilon_{1}^{h_{l}\left(\widehat{\sigma}_{k}^{S}, y\right)}\left(1-\varepsilon_{1}\right)^{m-h_{l}\left(\widehat{\sigma}_{k}^{S}, y\right)}} \\
& =\left(\frac{\varepsilon_{1}}{1-\varepsilon_{1}}\right)^{h_{l}\left(\widehat{\sigma}_{l}^{S}, y\right)+h_{k}\left(\widehat{\sigma}_{l}^{S}, y\right)-m}
\end{aligned}
$$


Ratio $_{3}$ : Refers to the probability of transformation of the 1's bits in the remaining blocks (all the blocks but $l$ and $k$ ), then, using lemma lemma2, part 1

$$
\text { Ratio }_{3}=\prod_{\alpha \neq l, k}^{|\Omega|} \frac{\varepsilon_{1}^{h_{\alpha}\left(\widehat{\sigma}_{l}^{S}, y\right)}\left(1-\varepsilon_{1}\right)^{m-h_{\alpha}\left(\widehat{\sigma}_{l}^{S}, y\right)}}{\varepsilon_{1}^{h_{\alpha}\left(\widehat{\sigma}_{k}^{S}, y\right)}\left(1-\varepsilon_{1}\right)^{m-h_{\alpha}\left(\widehat{\sigma}_{k}^{S}, y\right)}}=1
$$

Putting these three ratios together, we have that

$$
\begin{aligned}
\frac{p\left(\widehat{\sigma}_{l}^{S} \mid y\right)}{p\left(\widehat{\sigma}_{k}^{S} \mid y\right)} & =\frac{q_{l}}{q_{k}}\left(\frac{\varepsilon_{0}}{1-\varepsilon_{0}}\right)^{h_{l}\left(\widehat{\sigma}_{l}^{S}, y\right)+h_{k}\left(\widehat{\sigma}_{l}^{S}, y\right)-m}\left(\frac{\varepsilon_{1}}{1-\varepsilon_{1}}\right)^{h_{l}\left(\widehat{\sigma}_{l}^{S}, y\right)+h_{k}\left(\widehat{\sigma}_{l}^{S}, y\right)-m} \\
& =\frac{q_{l}}{q_{k}}\left(\frac{\varepsilon_{0}}{1-\varepsilon_{0}} \frac{\varepsilon_{1}}{1-\varepsilon_{1}}\right)^{h_{l}\left(\widehat{\sigma}_{l}^{S}, y\right)+h_{k}\left(\widehat{\sigma}_{l}^{S}, y\right)-m}
\end{aligned}
$$

and the proposition holds.

\section{Proof of the Theorem 1:}

The Receiver's condition: Given the Sender equilibrium strategy $\left\{\widehat{\sigma}_{j}^{S}\right\}_{j}$, and the Receiver's information set $Y^{n}$, consider the realization $y \in Y^{n}$. The Receiver's strategy is defined by: $\widehat{\sigma}_{y}^{R}=\widehat{a}_{l} \Leftrightarrow y \in Y_{l}$, where for each $l \in\{1, \ldots,|\Omega|\}$, and $\tilde{\Omega}_{l}=\left\{k \in\{1, \ldots,|\Omega|\}\right.$ such that $q_{l} M_{l} \neq$ $\left.q_{k} M_{k}\right\}$,

$$
\begin{aligned}
Y_{l}=\left\{y \in Y^{n} \mid \quad\right. & \left.\left.h_{l}\left(\widehat{\sigma}_{l}^{S}, y\right)\right)+h_{k}\left(\widehat{\sigma}_{l}^{S}, y\right)\right) \leq C_{l k}, \text { for all } k \in \tilde{\Omega}_{l} \\
& \left.\left.h_{l}\left(\widehat{\sigma}_{l}^{S}, y\right)\right)+h_{k^{\prime}}\left(\widehat{\sigma}_{l}^{S}, y\right)\right) \leq C_{l k^{\prime}}, \text { for all } k^{\prime} \epsilon \notin \tilde{\Omega}_{l}, k^{\prime}<l \\
& \left.\left.\left.h_{l}\left(\widehat{\sigma}_{l}^{S}, y\right)\right)+h_{k^{\prime}}\left(\widehat{\sigma}_{l}^{S}, y\right)\right)<C_{l k^{\prime}} \text {, for all } k^{\prime} \notin \tilde{\Omega}_{l}, k^{\prime}>l\right\}
\end{aligned}
$$

Parameters $\left\{C_{l k}\right\}_{l \neq k}$ are given by:

$$
C_{l k}=\frac{\operatorname{Ln} \frac{M_{k} q_{k}}{M_{l} q_{l}}}{\operatorname{Ln} \frac{\varepsilon_{0}}{1-\varepsilon_{0}} \frac{\varepsilon_{1}}{1-\varepsilon_{1}}}+m
$$

with associated payoff $\left.\pi_{y}^{R}\left(\left\{\widehat{\sigma}_{j}^{S}\right\}_{j}, \widehat{a}_{l}\right)=\sum_{j=1}^{|\Omega|} p\left(\widehat{\sigma}_{j}^{S} \mid y\right) u\left(\widehat{a}_{l}, \omega_{j}\right)=p\left(\widehat{\sigma}_{l}^{S}\right) \mid y\right) M_{l}$.

Consider any other strategy $a_{\alpha} \neq \widehat{a}_{l}$ and suppose that its associated payoff is higher than under $\widehat{a}_{l}$. Then, $\pi_{y}^{R}\left(\left\{\widehat{\sigma}_{j}^{S}\right\}_{j}, a_{\alpha}\right)>\pi_{y}^{R}\left(\left\{\widehat{\sigma}_{j}^{S}\right\}_{j}, \widehat{a}_{l}\right)$, or

$$
\sum_{j=1}^{|\Omega|} p\left(\widehat{\sigma}_{j}^{S} \mid y\right) u\left(\widehat{a}_{\alpha}, \omega_{j}\right)>\sum_{j=1}^{|\Omega|} p\left(\widehat{\sigma}_{j}^{S} \mid y\right) u\left(\widehat{a}_{l}, \omega_{j}\right),
$$

which by the linearity of $\pi_{y}^{R}$ in probabilities $p\left(\widehat{\sigma}_{j}^{S} \mid y\right)$, is equal to,

$$
p\left(\widehat{\sigma}_{\alpha}^{S} \mid y\right) M_{\alpha}>p\left(\widehat{\sigma}_{l}^{S} \mid y\right) M_{l}, \text { or } \quad \frac{M_{\alpha}}{M_{l}}>\frac{p\left(\widehat{\sigma}_{l}^{S} \mid y\right)}{p\left(\widehat{\sigma}_{\alpha}^{S} \mid y\right)}
$$


By Proposition 1, these inequalities can be written as

$$
\frac{q_{\alpha} M_{\alpha}}{q_{l} M_{l}}>\left(\frac{\varepsilon_{0}}{1-\varepsilon_{0}} \frac{\varepsilon_{1}}{1-\varepsilon_{1}}\right)^{h_{l}\left(\widehat{\sigma}_{l}^{S}, y\right)+h_{\alpha}\left(\widehat{\sigma}_{l}^{S}, y\right)-m}
$$

We write this condition with the Logarithm operator:

$$
\operatorname{Ln} \frac{q_{\alpha} M_{\alpha}}{q_{l} M_{l}}>\left(h_{l}\left(\widehat{\sigma}_{l}^{S}, y\right)+h_{\alpha}\left(\widehat{\sigma}_{l}^{S}, y\right)-m\right) \operatorname{Ln} \frac{\varepsilon_{0}}{1-\varepsilon_{0}} \frac{\varepsilon_{1}}{1-\varepsilon_{1}}
$$

Since $\varepsilon_{0}+\varepsilon_{1}<1$, then $\frac{\varepsilon_{0}}{1-\varepsilon_{0}} \frac{\varepsilon_{1}}{1-\varepsilon_{1}}<1$ and $\ln \left(\frac{\varepsilon_{0}}{1-\varepsilon_{0}} \frac{\varepsilon_{1}}{1-\varepsilon_{1}}\right)<0$. Then, the above inequality is equivalent to:

$$
h_{l}\left(\widehat{\sigma}_{l}^{S}, y\right)+h_{\alpha}\left(\widehat{\sigma}_{l}^{S}, y\right)>\frac{\operatorname{Ln} \frac{q_{\alpha} M_{\alpha}}{q_{l} M_{l}}}{\operatorname{Ln} \frac{\varepsilon_{0}}{1-\varepsilon_{0}} \frac{\varepsilon_{1}}{1-\varepsilon_{1}}}+m=C_{l \alpha}
$$

by the definition of $C_{l \alpha}$. But this contradicts that $y \in Y_{l}$, since by definition $\left.Y_{l}=\left\{y \in Y^{n} \mid h_{l}\left(\sigma_{l}^{S}, y\right)\right)+h_{k}\left(\sigma_{l}^{S}, y\right)\right) \leq C_{l k}$, for all $\left.k \neq l\right\}$, in particular for $k=\alpha$. Therefore for each $y \in Y^{n}$ there is no profitable deviation from $\widehat{\sigma}_{y}^{R}$, and $\widehat{\sigma}_{y}^{R}$ is a best response to $\left\{\widehat{\sigma}_{j}^{S}\right\}_{j}$.

The Sender's condition. Truth-telling: The Sender's strategy at state $\omega_{j}$ consists of sending a message and thus it suffices to show that there is no profitable deviation by sending another message different from $\widehat{\sigma}_{j}^{S}$, when $R$ plays $\left\{\widehat{\sigma}_{y}^{R}\right\}_{y}$. The associated payoff of $\widehat{\sigma}_{j}^{S}$ at state $\omega_{j}$ when the Receiver plays his equilibrium strategy $\left\{\widehat{\sigma}_{y}^{R}\right\}_{y}$ is

$$
\pi_{j}^{S}\left(\widehat{\sigma}_{j}^{S},\left\{\widehat{\sigma}_{y}^{R}\right\}_{y}\right)=\sum_{y \in Y^{n}} p\left(y \mid \widehat{\sigma}_{j}^{S}\right) u\left(\widehat{\sigma}_{y}^{R}, \omega_{j}\right)=M_{j} \sum_{y \in Y_{j}} p\left(y \mid \widehat{\sigma}_{j}^{S}\right)
$$

since $u\left(a_{t}, \omega_{j}\right)=0$ for all $a_{t} \neq \widehat{a}_{j}$.

Consider the associated payoff of sending any other message $x \in X^{n}$,

$$
\sum_{y \in Y^{n}} p(y \mid x) u\left(\widehat{\sigma}_{y}^{R}, \omega_{j}\right)=M_{j} \sum_{y \in Y_{j}} p(y \mid x)
$$

Let $f=h\left(\widehat{\sigma}_{j}^{S}, x\right)$ be the Hamming distance between messages $\widehat{\sigma}_{j}^{S}$ and $x$. We can construct a sequence of messages $\left\{\theta_{0}, \theta_{1}, \ldots, \theta_{f}\right\}$ such that $\theta_{i} \in X^{n}, \theta_{0}=\widehat{\sigma}_{j}^{S}, \theta_{f}=x$ satisfying that, for all $d=0, \ldots, f-1$,

$$
h\left(\widehat{\sigma}_{j}^{S}, \theta_{d+1}\right)=h\left(\widehat{\sigma}_{j}^{S}, \theta_{d}\right)+1 \text { and } h\left(\theta_{d}, \theta_{d+1}\right)=1
$$

This sequence transforms message $\widehat{\sigma}_{j}^{S}$ into message $x$ by only changing one element at each step. Let us show that, for all $d=0, \ldots, f-1$,

$$
\sum_{y \in Y_{j}} \frac{p\left(y \mid \theta_{d}\right)}{p\left(y \mid \theta_{d+1}\right)} \geq 1
$$


Let $i_{d}$ be the location of the (unique) mismatch between $\theta_{d}$ and $\theta_{d+1}$. Then,

$$
\begin{aligned}
\sum_{y \in Y j} \frac{p\left(y \mid \theta_{d}\right)}{p\left(y \mid \theta_{d+1}\right)} & =\sum_{y \in Y_{j}} \frac{\prod_{i=1, \ldots, n} p\left(y^{i} \mid \theta_{d}^{i}\right)}{\prod_{i=1, \ldots, n} p\left(y^{i} \mid \theta_{d+1}^{i}\right)}=\sum_{y \in Y_{j}} \frac{p\left(y^{i_{d}} \mid \theta_{d}^{i_{d}}\right)}{p\left(y^{i_{d}} \mid \theta_{d+1}^{i_{d}}\right)} \\
& =\sum_{y \in Y_{j} \mid y^{i} d=0} \frac{p\left(0 \mid \theta_{d}^{i_{d}}\right)}{p\left(0 \mid \theta_{d+1}^{i_{d}}\right)}+\sum_{y \in Y_{j} \mid y^{i} d=1} \frac{p\left(1 \mid \theta_{d}^{i_{d}}\right)}{p\left(1 \mid \theta_{d+1}^{i_{d}}\right)}
\end{aligned}
$$

Let us consider two different cases:

Case 1: $(j-1) m-1 \leq i_{d} \leq j m$. The mismatch occurs at block $j$ and as $y \in Y_{j}$, then the element $\theta_{d}^{i_{d}}$ coincides with the element $y^{i_{d}}=0$ and $\theta_{d+1}^{i_{d}}=1$. The above expression is now given by:

$$
\begin{aligned}
\sum_{y \in Y_{j}} \frac{p\left(y \mid \theta_{d}\right)}{p\left(y \mid \theta_{d+1}\right)} & =\sum_{y \in Y_{j} \mid y^{i} d=0} \frac{p(0 \mid 0)}{p(0 \mid 1)}+\sum_{y \in Y_{j} \mid y^{i} d=1} \frac{p(1 \mid 0)}{p(1 \mid 1)} \\
& =\sum_{y \in Y_{j} \mid y^{i} d=0} \frac{1-\varepsilon_{0}}{\varepsilon_{1}}+\sum_{y \in Y_{j} \mid y^{i} d=1} \frac{\varepsilon_{0}}{1-\varepsilon_{1}} \geq 1
\end{aligned}
$$

Notice that there exists at least an element $y \in Y_{j}$ with $y^{i_{d}}=0$ and the ratio $\frac{1-\varepsilon_{0}}{\varepsilon_{1}} \geq 1$ because $\varepsilon_{0}+\varepsilon_{1}<1$. Therefore $\sum_{y \in Y_{j} \mid y^{i} d=0} \frac{1-\varepsilon_{0}}{\varepsilon_{1}} \geq 1$.

Case 2: $i_{d}<(j-1) m-1$ or $i_{d}>j m$. The mismatch occurs in a different block of $j$ and as above $y \in Y_{j}$, then the element $\theta_{d}^{i_{d}}$ coincides with the element $y^{i_{d}}=1$ and $\theta_{d+1}^{i_{d}}=0$. The above expression is now given by:

$$
\begin{aligned}
\sum_{y \in Y_{j}} \frac{p\left(y \mid \theta_{d}\right)}{p\left(y \mid \theta_{d+1}\right)} & =\sum_{y \in Y_{j} \mid y^{i} d=0} \frac{p(0 \mid 1)}{p(0 \mid 0)}+\sum_{y \in Y_{d} \mid y^{i} d=1} \frac{p(1 \mid 1)}{p(1 \mid 0)} \\
& =\sum_{y \in Y_{j} \mid y^{i} d=0} \frac{\varepsilon_{1}}{1-\varepsilon_{0}}+\sum_{y \in Y_{j} \mid y^{i} d=1} \frac{1-\varepsilon_{1}}{\varepsilon_{0}} \geq 1
\end{aligned}
$$

The set of elements in $Y_{j}$ such that $y^{i_{d}}=1$ has cardinality greater than or equal to 1 . Therefore $\sum_{y \in Y_{j} \mid y^{i} d=1} \frac{1-\varepsilon_{1}}{\varepsilon_{0}} \geq 1$. From the above reasoning, the probability $p\left(y \mid \theta_{d}\right)$ decreases at each step of the deviation chain $\left\{\widehat{\sigma}_{j}^{S}, \theta_{1}, \ldots, x\right\}$. We conclude that $\sum_{y \in Y_{j}} p\left(y \mid \widehat{\sigma}_{j}^{S}\right) \geq \sum_{y \in Y_{j}} p(y \mid x)$ and the associated payoffs for both messages $\widehat{\sigma}_{j}^{S}$ and $x$ verify the condition $M_{j} \sum_{y \in Y_{j}} p\left(y \mid \widehat{\sigma}_{j}^{S}\right) \geq M_{j} \sum_{y \in Y_{j}} p(y \mid x)$ that closes the proof. Hence, for each $\widehat{\sigma}_{j}^{S}$ is a best response to $\left\{\widehat{\sigma}_{y}^{R}\right\}_{y}$.

Since at each state $\omega_{j}$, the Sender pure strategy $\widehat{\sigma}_{j}^{S}$ is a best response to $\left\{\widehat{\sigma}_{y}^{R}\right\}_{y}$ and for each $y \in Y^{n}$, the Receiver pure strategy $\widehat{\sigma}_{y}^{R}$ is a best response to $\left\{\widehat{\sigma}_{j}^{S}\right\}_{j}$, then the pair of tuples $\left(\left\{\widehat{\sigma}_{j}^{S}\right\}_{j},\left\{\widehat{\sigma}_{y}^{R}\right\}_{y}\right)$ is a pure strategy Nash equilibrium of $\Gamma_{v}^{n}$. 
Proof of proposition 2: Without loss of generality, let us assume that $j=1$ and $C_{12} \leq C_{13} \leq \ldots \leq C_{1|\Omega|}$. It suffices to be proven that:

$$
p\left(Y_{1}^{n} \mid \widehat{\sigma}_{1}^{S}\right) \geq 1-\varepsilon_{1}^{c_{12}+1} \phi\left(\varepsilon_{0}, \varepsilon_{1}\right)
$$

where $\phi\left(\varepsilon_{0}, \varepsilon_{1}\right)$ is a function on $\varepsilon_{0}$ and $\varepsilon_{1}$ such that $0 \leq \phi\left(\varepsilon_{0}, \varepsilon_{1}\right) \leq 1$. The probability $p\left(Y_{1}^{n} \mid \widehat{\sigma}_{1}^{S}\right)$ can be written as

$$
p\left(Y_{1}^{n} \mid \widehat{\sigma}_{1}^{S}\right)=1-p\left(Y^{n}-Y_{1}^{n} \mid \widehat{\sigma}_{1}^{S}\right)
$$

Let $J \subseteq\{2,3, \ldots,|\Omega|\}$ and let us $E_{J}$ denote the event $E_{J}=\left\{y \in Y^{n}-Y_{1}^{n} \mid h_{1}\left(\widehat{\sigma}_{1}^{S}, y\right)+h_{j}\left(\widehat{\sigma}_{1}^{S}, y\right)>C_{1 j}\right.$ if $j \in J$ and $h_{1}\left(\widehat{\sigma}_{1}^{S}, y\right)+h_{l}\left(\widehat{\sigma}_{1}^{S}, y\right) \leq C_{1 l}$ if $l \notin J$

We have that:

$$
\begin{aligned}
E_{J_{1}} \cap E_{J_{2}} & =\emptyset \text { if } J_{1}, J_{2} \subseteq\{2,3, \ldots,|\Omega|\}, J_{1} \neq J_{2} \\
Y^{n}-Y_{1}^{n} & =\bigcup_{\substack{J \subseteq\{2,3, \ldots,|\Omega|\} \\
J \neq \emptyset}} E_{J}
\end{aligned}
$$

In other words, $\left\{E_{J}\right\}_{J \subseteq\{2,3, \ldots,|\Omega|\}}$ is a partition of $Y^{n}-Y_{1}^{n}$ formed by disjoint events. In such a case, the probability $p\left(y \in Y^{n}-Y_{1}^{n} \mid \widehat{\sigma}_{1}^{S}\right)$ can be written as:

$$
p\left(y \in Y^{n}-Y_{1}^{n} \mid \widehat{\sigma}_{1}^{S}\right)=p\left(\bigcup_{\substack{J \subseteq\{2,3, \ldots,|\Omega|\} \\ J \neq \emptyset}} E_{J} \mid \widehat{\sigma}_{1}^{S}\right)=\sum_{\substack{J \subseteq\{2,3, \ldots,|\Omega|\} \\ J \neq \emptyset}} p\left(E_{J} \mid \widehat{\sigma}_{1}^{S}\right)
$$

Similarly, for each $J \subseteq\{2,3, . .|\Omega|\}$, let $y_{J} \in E_{J}$ such that $h_{1}\left(\widehat{\sigma}_{1}^{S}, y_{J}\right)+$ $h_{j}\left(\widehat{\sigma}_{1}^{S}, y_{J}\right)=c_{1 j}+1$.

Since

$$
\begin{aligned}
& p\left(y_{J} \mid \widehat{\sigma}_{1}^{S}\right)=\varepsilon_{0}^{h_{1}\left(\widehat{\sigma}_{1}^{S}, y_{J}\right)} \varepsilon_{1}^{\left.h_{2}\left(\widehat{\sigma}_{1}^{S}, y_{J}\right)+\ldots+h_{|\Omega|} \mid \widehat{\sigma}_{1}^{S}, y_{J}\right)} \leq \varepsilon_{1}^{h_{1}\left(\widehat{\sigma}_{1}^{S}, y_{J}\right)+h_{2}\left(\widehat{\sigma}_{1}^{S}, y_{J}\right)}=\varepsilon_{1}^{c_{12}+1} \text {, then } \\
& p\left(Y^{n}-Y_{1}^{n} \mid \widehat{\sigma}_{1}^{S}\right)= \sum_{\substack{J \subseteq\{2,3, \ldots,|\Omega|\} \\
J \neq \emptyset}} p\left(E_{J} \mid y_{J}, \widehat{\sigma}_{1}^{S}\right) p\left(y_{J} \mid \widehat{\sigma}_{1}^{S}\right) \\
& \leq \varepsilon_{1}^{c_{12}+1} \sum_{\substack{J \subseteq\{2,3, \ldots,|\Omega|\} \\
J \neq \emptyset}} p\left(E_{J} \mid y_{J}, \widehat{\sigma}_{1}^{S}\right)=\varepsilon_{1}^{c_{12}+1} p(\underbrace{}_{\substack{J \subseteq\{2,3, \ldots,|\Omega|\} \\
J \neq \emptyset}} E_{J} \mid y_{J}, \widehat{\sigma}_{1}^{S})
\end{aligned}
$$


Let

$$
\begin{gathered}
\phi\left(\varepsilon_{0}, \varepsilon_{1}\right)=p\left(\bigcup_{\substack{J \subseteq\{2,3, \ldots,|\Omega|\} \\
J \neq \emptyset}} E_{J} \mid y_{J}, \widehat{\sigma}_{1}^{S}\right), \text { then } \\
p\left(Y_{1}^{n} \mid \widehat{\sigma}_{1}^{S}\right) \geq 1-\varepsilon_{1}^{c_{12}+1} \phi\left(\varepsilon_{0}, \varepsilon_{1}\right)
\end{gathered}
$$

and the theorem holds. 